JOURNAL OF THE

AMERICAN MATHEMATICAL SOCIETY

Volume 17, Number 4, Pages 749-782

S 0894-0347(04)00463-1

Article electronically published on August 27, 2004

\title{
REAL BOUNDS, ERGODICITY AND NEGATIVE SCHWARZIAN FOR MULTIMODAL MAPS
}

\author{
SEBASTIAN VAN STRIEN AND EDSON VARGAS
}

\section{INTRODUCTION AND STATEMENT OF RESULTS}

Over the last 20 years, many of the most spectacular results in the field of dynamical systems dealt specifically with interval and circle maps (or perturbations and complex extensions of such maps). Primarily, this is because in the one-dimensional case, much better distortion control can be obtained than for general dynamical systems. However, many of these spectacular results were obtained so far only for unimodal maps. The aim of this paper is to provide all the tools for studying general multimodal maps of an interval or a circle, by obtaining

- real bounds controlling the geometry of domains of certain first return maps, and providing a new (and we believe much simpler) proof of absense of wandering intervals;

- provided certain combinatorial conditions are satisfied, large real bounds implying that certain first return maps are almost linear;

- Koebe distortion controlling the distortion of high iterates of the map, and negative Schwarzian derivative for certain return maps (showing that the usual assumption of negative Schwarzian derivative is unnecessary);

- control of distortion of certain first return maps;

- ergodic properties such as sharp bounds for the number of ergodic components.

We will give historical comments below the statements of the theorems.

There are many applications and potential applications of our bounds. For example, it is clear that any future renormalization results for multimodal maps (generalizing the unimodal results of Sullivan, McMullen, Lyubich, de Melo, Avila ...) would require real bounds. Our real bounds are one of the key ingredients in the proof that Axiom A maps are dense within the space of real polynomials with real critical points; see [7.

Let us now be more precise. Let $M=[-1,1]$ or $M=S^{1}$, and let $f: M \rightarrow M$ be a smooth map. This map is called multimodal if $M$ has a partition into finitely many subintervals on which $f$ is strictly monotone. Without loss of generality, we may and will assume that $f(\partial M) \subset \partial M$. Let $c_{1}, \ldots, c_{d}$ be the critical points of $f$,

Received by the editors May 1, 2002.

2000 Mathematics Subject Classification. Primary 37Exx, 37Fxx.

Key words and phrases. Dynamical systems, interval dynamics, holomorphic dynamics.

The first author was partially supported by EPSRC grant GR/R73171/01.

The second author was partially supported by CNPq-Brasil, Grant \#300557/89-2(RN). 
i.e., the points where $f^{\prime}$ is zero. Throughout this paper we will assume that $f$ is $C^{k}$ outside these critical points, and that $f$ is non-flat at these critical point, i.e., that for $i=1, \ldots, d$ and $x$ near $c_{i}$ we can write

$$
f(x)= \pm\left|\phi_{i}(x)\right|^{\beta_{i}}+f\left(c_{i}\right),
$$

where $\phi_{i}$ is $C^{k}, \phi_{i}\left(c_{i}\right)=0$ and $\beta_{i}>1$. Here $k=2$ is enough for Theorems A, B(1) and $\mathrm{C}(1)$, and $k=3$ is enough for the remaining theorems. In fact, for Theorems $\mathrm{A}, \mathrm{B}(1)$ and $\mathrm{C}(1)$ it suffices to take $k=1+Z$ ygmund; see [17. We denote the class of such maps by $\mathcal{A}^{k}$ and note that $\mathcal{A}^{3} \subset \mathcal{A}^{2} \subset \mathcal{A}^{1+Z y g m u n d}$.

If $\mathcal{B}$ is a Borelean set, we will denote its Lebesgue measure by $|\mathcal{B}|$. We will also use the following two definitions:

Definition 1. Let $U, V$ be bounded intervals such that the closure of $U$ is contained in the interior of $V$. We say that $V$ is an $\alpha$-scaled neighbourhood of $U$ if $\left|U^{+}\right| \geq \alpha|U|$ and $\left|U^{-}\right| \geq \alpha|U|$, where $U^{+}$and $U^{-}$are the connected components of $V \backslash U$. We also sometimes say that $U$ is $\alpha$-well-inside $V$.

Definition 2. An open interval $I \subset[-1,1]$ is called a nice interval if the forward orbit of its boundary does not intersect $I$; that is, $I \cap f^{i}(\partial I)=\emptyset$ for each $i \geq 0$.

One reason to consider the concept of nice intervals is the fact that the domain of the first return map of a nice interval $I$ consists of a countable union of open intervals, called return domains, whose boundaries are mapped into the boundary of $I$. Moreover, two intervals in the backward orbit of a nice interval are nested or disjoint. Intervals of the backward orbit of a nice interval are nice intervals too.

Let us now define a sequence of nice intervals around any point $x \in M$. Let $I_{0}=I$ be a nice interval containing $x$. In the Yoccoz partition, it is customary to choose $I$ to be an interval of the backward orbit of a component of $M \backslash f^{-1}(P)$ where $P$ is the set of fixed points of $f$, and then you can take for $I_{0}$ the component of $M \backslash f^{-1}(P)$ containing $x$. But any other choice for $I_{0}$ is also possible in this paper. Next define inductively a sequence of nice intervals $I_{n}$ containing $x$ as follows. Let $\phi_{n}$ be the first return map to $I_{n-1}$, and let $I_{n}$ be the domain of $\phi_{n}$ containing $x$. If $x$ is not in the domain of $\phi_{n}$, then we define $I_{n}=I_{n+1}=\cdots=\emptyset$. Of course, if $x$ is recurrent, then $I_{n} \neq \emptyset$ and if $I_{0}$ is periodic, $I_{n}=I_{0}$. We say that $\phi_{n+1}: I_{n+1} \rightarrow I_{n}$ is non-central w.r.t. $x \in I_{n+1}$ if $\phi_{n+1}(x) \notin I_{n+1}$. We say that $f$ has real bounds at $x$ if there exists $\xi>0$ such that $I_{n+1}$ is $\xi$-well-inside $I_{n}$ whenever $\phi_{n}: I_{n} \rightarrow I_{n-1}$ is non-central. By the second part of Theorem A, then all domains of the first return map to $I_{n+1}$ are $\xi^{\prime}$-well-inside $I_{n+1}$.

1.1. Real bounds. Our first theorem controls the geometry of the domains of first return maps to nice intervals and can be used to show that first return maps to arbitrarily small neighbourhoods of $x$ are well-controlled.

Theorem A (Real bounds). For each $f \in \mathcal{A}^{1+Z y g m u n d}$, there exist $\xi_{0}>0$ and a function $\rho: \mathbb{R}^{+} \rightarrow \mathbb{R}^{+}$such that if $x \in M$ and $\phi_{1}: I_{1} \rightarrow I_{0}$ is non-central w.r.t. $x$, then the following hold.

(1) If $\phi_{n}: I_{n} \rightarrow I_{n-1}$ is non-central w.r.t. $x$ and $n \geq 2$, then the interval $I_{n+1}$ is $\xi_{0}$-well-inside $I_{n}$.

(2) If $I_{n+1}$ is $\xi$-well-inside $I_{n}$, then all domains of the first return map to $I_{n+1}$ are $\xi^{\prime}=\rho(\xi)$-well-inside $I_{n+1}$. 
We do not assume that $x$ is recurrent, but note that if the interval $I_{n+1}$ is empty, then we consider the statement in Theorem A to be trivially true. Let us also remark that for any map $f$ and any integer $n$, if $x$ is a turning point of $f$, then either $I_{n+1}$ is well-inside $I_{n}$ or there exists a definite neighbourhood of $I_{n}$ that one can pull back to $I_{n+1}$ with bounded intersection multiplicity; see Lemma 2 .

The constant $\xi_{0}$ and the function $\rho$ in the previous theorem are universal: they only depend on the constant $\gamma(f)$ associated to $f$ defined in equations (1) and (2) in Section 2. We note that $\gamma(f)$ does not depend on the dynamics of $f$, but only on the $C^{2}$ norm of $f$, the number of critical points and their order.

We need to assume in part (1) of the previous theorem that $n \geq 2$ (it is easy to give a counterexample if $n$ is allowed to be equal to 1 ).

We should emphasize that we do not use the non-existence of wandering intervals in the proof of Theorem A. In fact, we obtain an independent proof for the nonexistence of wandering intervals:

Corollary of the proof of Theorem A (Non-existence of wandering intervals). Let $f \in \mathcal{A}^{1+Z y g m u n d}$ and assume that $J \subset M$ is an interval for which all iterates $J, f(J), \ldots$ are mutually disjoint. Then $\left\{f^{n}(J)\right\}_{n \geq 0}$ converges to an (possibly onesided) attracting periodic orbit.

It is well known (and easy to show) that this implies the contraction principle: for each $\delta>0$ there exists $\epsilon>0$ so that if $J$ is an interval with $|J|<\epsilon$ and not intersecting the immediate basin of a periodic attractor, then each component of $f^{-n}(J)$ has length $\leq \delta$.

If for all $n$ larger than some $n_{0}, \phi_{n+1}: I_{n+1} \rightarrow I_{n}$ is central w.r.t. $x$, then $x$ is contained in the periodic interval $\bigcap I_{n}$ and (if $\bigcap I_{n}$ is not a singleton) $f$ is said to be renormalizable (at $x$ ). Of course, in this case one can again apply Theorem $\mathrm{A}$ to any nice subinterval of the periodic interval $\bigcap I_{n}$. So even if $f$ is (infinitely often) renormalizable, Theorem A is relevant. To be more precise,

Theorem $\mathbf{A}^{\prime}$ (Real bounds on arbitrarily small scales). For each $f \in \mathcal{A}^{1+Z y g m u n d}$ there exists $\xi_{0}>0$ so that for any non-periodic point $x \in M$ that is not in the basin of a periodic attractor, either (1) or (2) holds.

(1) $f$ is infinitely renormalizable at $x$ of Feigenbaum-type: there exist an integer $p$ and nice periodic intervals $K_{n} \ni x$ of period $p 2^{n}$ for every $n \geq 0$ such that $K_{n+1}$ is $\xi_{0}$-well-inside $K_{n}$. ( $K_{n}$ is nice, so $f^{p 2^{n}}\left(\partial K_{n}\right) \subset \partial K_{n}$.)

(2) There are arbitrarily small nice intervals $I$ around $x$ such that the return domain $J$ to $I$ containing $x$ is $\xi_{0}$-well inside $I$.

Moreover, define in case (1) $I=K_{n}$ and $J=K_{n+1}$, and in case (2) $I \supset J:=$ $\mathcal{L}_{x}(I)$. Then $I, J$ are both nice intervals. If $I_{i} \supset J_{i}$ and $I_{j} \supset J_{j}$ are pullbacks of $I \supset J$, then they are either nested or disjoint, and if $J_{i} \subset I_{j}$, then $I_{i} \subset I_{j}$.

Here, and throughout this paper, we denote by $\mathcal{L}_{x}(I)$ the component containing $x$ of the domain of the first entry map to $I$.

Real bounds as in Theorem A, but around recurrent turning points, were proved previously by Martens in the negative Schwarzian unimodal case and by Vargas in the case of $C^{2}$ multimodal maps without inflection points; see [14], [23] and also Shen's paper [18]. If all branches of $f$ are monotone and there is at most one critical point (of inflection type), then such bounds were proved by Levin; see [8] and also [10]. Levin's proof (in particular Proposition 4 of his paper) does not seem to work 
if there are several critical points. The reason that inflection points complicate matters considerably is that when a return domain $J$ to an interval $I$ is very small compared to $I$, it still need not be well-inside $I$. If $J$ contains a turning point, one has symmetry, and this problem does not arise. Even if there are no inflection points, our proof is quite different from previous proofs.

Non-existence of wandering intervals for one-dimensional maps was proved in a series of papers by Denjoy, Guckenheimer, Yoccoz, de Melo \& van Strien, Blokh \& Lyubich, Martens \& de Melo \& van Strien. For historical references, see [17.

1.2. Large bounds. In applications one often needs large real bounds, in particular for proving density of Axiom A; see [20] and [7]. If $I$ is a nice interval containing a non-periodic recurrent critical point $c$, then we say that $J$ is a child of $I$ if $J$ is a component of $f^{-1}\left(J^{\prime}\right)$ where $J^{\prime}$ is a diffeomorphic pullback of $I$, and $J$ contains a critical point $c^{\prime}$ with $\omega(c)=\omega\left(c^{\prime}\right) \ni c, c^{\prime}$. If $J_{1}, J_{2}$ are two children of $I$, then they are either disjoint or they lie nested. In the next theorem we give two ways in which one can get large bounds. Parts (1) and (2) of Theorem B were proved previously by Weixiao Shen in, respectively, [18] and [20, Proposition 4.1].

Theorem B (Large bounds). For any $f: M \rightarrow M$ in the class $\mathcal{A}^{3}$ the following hold.

(1) For each $C>0, \delta>0$ there exists $N^{\prime}$ so that if a nice interval $I$ has at least $N^{\prime}$ children $K_{1} \supset K_{2} \supset \ldots$ containing some critical point, and each of its return domains to $I$ is $\delta$-well-inside $I$, then for $I^{\prime}=K_{N}$ with $N \geq N^{\prime}$ each of its return domains to $I^{\prime}$ is $C$-well-inside $I^{\prime}$.

(2) There exists a function $\rho: \mathbb{R}^{+} \rightarrow \mathbb{R}^{+}$with $\rho(\xi) \rightarrow \infty$ as $\xi \rightarrow \infty$ such that if $I_{n+1}$ is $\xi$-well-inside $I_{n}$, then each return domain to $I_{n+1}$ is $\rho(\xi)$-well-inside $I_{n+1}$.

The proof of Theorem $\mathrm{B}(1)$ also shows that if $x$ is a recurrent point and $\omega(x)$ is a non-minimal set, then one can find for each $\xi>0$, a nice interval $I$ around $x$ such that the component of the first return map containing $x$ is $\xi$-well-inside $I$. (One can also argue as in the proof of Theorem $\mathrm{A}^{\prime}$ in [10.) (That a set $X$ is minimal means that each orbit in $X$ is dense in $X$.)

As before, $N^{\prime}$ and $\rho$ only depend on $\gamma(f)$ from equations (1) and (2) in Section 2 and not on $f$.

1.3. Koebe and negative Schwarzian. One of the reasons real bounds are so useful is because of the following distortion theorem. Parts (1) and (2) show that, surprisingly, one can often use Koebe even if one does not have disjointness of intervals.

Theorem C (Koebe and negative Schwarzian). Let $f: M \rightarrow M$ be in the class $\mathcal{A}^{3}$. Then one has the following properties.

(1) [Improved Macroscopic Koebe Principle] For each $\xi>0$, there exists $\xi^{\prime}>$ 0 such that if $I$ is a nice interval, $V$ is $\xi$-well-inside $I$ and $x \in I$ and $f^{k}(x) \in V$ (with $k \geq 1$ not necessarily minimal), then the pullback of $V$ along $\left\{x, \ldots, f^{k}(x)\right\}$ is $\xi^{\prime}$-well-inside the return domain to $I$ containing $x$.

(2) [Improved Koebe Principle] For each $S>0, \delta>0$ and $\xi>0$ there exists $K>0$ such that if $J \subset T$ are intervals, with $f_{\mid T}^{n}$ a diffeomorphism, $f^{n}(J)$ $\xi$-well-inside $f^{n}(T)$ and either

(i) $\sum_{i=0}^{n-1}\left|f^{i}(J)\right| \leq S$ or 
(ii) $f^{n}(T) \cap B_{0}(f)=\emptyset$ and $\operatorname{dist}\left(f^{i}(T)\right.$, Par $) \geq \delta, i=0, \ldots, n-1$, then $f_{\mid J}^{n}$ has bounded distortion, i.e., for any $x, y \in J$,

$$
\left|D f^{n}(x)\right| /\left|D f^{n}(y)\right| \leq K .
$$

Here $B_{0}(f)$ is the union of the immediate basins of the periodic (possibly parabolic) attractors and $\mathbf{P a r}$ is the set of parabolic periodic points of $f$.

(3) [Negative Schwarzian Derivative] For each critical point $c_{i}$ that is not in the basin of a periodic attractor, there exists a neighbourhood $U_{i}$ such that whenever $f^{n}(x) \in U_{i}$ for some $x \in M$ and some $n \geq 0$, then the Schwarzian derivative of $f^{n+1}$ at $x$ is negative:

$$
S f^{n+1}(x)<0 .
$$

In part (1) no disjointness is required, and the pullback of $I$ along $\left\{x, \ldots, f^{k}(x)\right\}$ can even meet a critical point an arbitrarily large number of times! In fact, in part

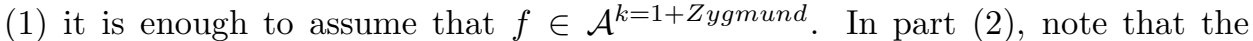
period of periodic attractors and parabolic orbits of $f$ is bounded; see [17. In part (2) one can choose $K$ close to 1 if $\xi$ is large.

The last part of Theorem $\mathrm{C}$ generalizes the results of Kozlovski [6] (which he proved for unimodal maps) to general multimodal maps. Since this paper was written, we have learned that Duncan Sands has used our bounds to obtain the following version of Theorem $\mathrm{C}(3)$ : if all periodic points are hyperbolic and repelling, then $f$ is conjugate to a map with negative Schwarzian derivative.

1.4. Distortion control of first entry maps. From Theorem $\mathrm{C}$ we get that first entry maps have bounded distortion:

Theorem D (Control of distortion). Assume that $f: M \rightarrow M$ is in the class $\mathcal{A}^{3}$. Then for each $\epsilon>0$ and for each critical point $c_{i}$ there exists a nice interval $V_{i}$ of diameter $<\epsilon$ such that $c_{i} \in V_{i}$ and so that the first entry map $\Psi$ to $\bigcup V_{i}$ is quasi-polynomial (more precisely, a composition of at most $d$ maps of the type $L_{1} \circ f \circ L_{2}$, where $L_{1}, L_{2}$ are diffeomorphisms of bounded distortion). If $f$ is infinitely renormalizable at $c_{i}$, then one can take for $V_{i}$ a periodic interval containing $c_{i}$.

In Section 8 a more precise version of this theorem will be stated. It is important to remark that the first return map to a single critical neighbourhood $V_{i_{0}}$ need not be 'quasi-polynomial' ( $\Psi$ is a composition of finitely many maps of type $L_{1} \circ f \circ$ $L_{2}$, where $L_{1}, L_{2}$ are diffeomorphisms of bounded distortion): the pullback of any definite neighbourhood of $V_{i}$ along a branch of the first return map to $V_{i_{0}}$ can meet a critical point an arbitrarily large number of times. So the situation is definitely much more complicated in the multimodal case than in the unimodal case.

1.5. Ergodic properties. For the next theorem we define a partial ordering on the set of critical points $\mathbf{C r}: c_{i} \prec c_{j}$ iff either $c_{i} \in \omega\left(c_{j}\right)$ or $c_{i}=c_{j}$. We then define $c_{i} \sim c_{k}$ iff both $c_{i} \prec c_{k}$ and $c_{k} \prec c_{i}$ (so in this case $\omega\left(c_{i}\right)=\omega\left(c_{k}\right)$ ).

Theorem E (Ergodic properties). For any $f: M \rightarrow M$ in the class $\mathcal{A}^{3}$ the following properties hold.

(1) Any minimal set $X$ has zero Lebesgue measure: for any point $x \in X$ there are intervals $\mathcal{N}_{n} \subset \mathcal{U}_{n}$ such that $\bigcap \mathcal{U}_{n}=\{x\},\left(\mathcal{U}_{n} \backslash \mathcal{N}_{n}\right) \cap X=\emptyset$ and $\mathcal{N}_{n}$ is $\xi$-well-inside $\mathcal{U}_{n}$, where $\xi>0$ does not depend on $n$. 
(2) There are finitely many compact forward invariant sets $X_{1}, \ldots, X_{k}$ such that $\bigcup B\left(X_{i}\right)$ has full measure in $M$. Here $B\left(X_{i}\right)$ is the basin of $X_{i}$ (i.e., $\left.B\left(X_{i}\right):=\left\{y ; \omega(y)=X_{i}\right\}\right)$. Moreover, either

- $X_{i}$ is an attracting periodic orbit,

- $X_{i}$ is a cycle of intervals containing a turning point and such that $\omega(x)=$ $X_{i}$ for a.e. $x \in X_{i}$, or

- $X_{i}$ is a minimal set containing at least one recurrent critical point.

(3) For any set $Y$ of positive Lebesgue measure with $f(Y) \subset Y$, not intersecting basins of periodic attractors and not containing intervals (up to measure zero), there exists a minimal set $X$ containing a critical point $c$ such that $|Y \cap B(X)|>0$ and such that for the intervals $\mathcal{U}_{n} \supset \mathcal{N}_{n} \ni c$ from part (1), $\left|Y \cap \mathcal{N}_{n}\right| /\left|\mathcal{N}_{n}\right| \rightarrow 1$.

(4) If $X_{i}$ is not an attracting periodic orbit, then $f_{\mid B\left(X_{i}\right)}$ is ergodic with respect the Lebesgue measure. The number of these $X_{i}$ 's is bounded by the number of equivalence classes of $\mathbf{C r} / \sim$ that are minimal w.r.t. $\prec$ (see the definition above).

From [17] it is known that the number of non-equivalent attracting periodic points is bounded. Here we say that two periodic points $p, q$ are equivalent if all iterates of $f$ are homeomorphisms on $(p, q)$.

Theorem E sharpens the classification of measure-theoretical attractors and ergodicity for smooth unimodal and multimodal maps obtained previously by Blokh and Lyubich; see [2] and 12].

\section{SOME OF THE TOOLS USED IN THIS PAPER}

Definition 3. A sequence $\left\{G_{i}\right\}_{i=0}^{l}$ of open intervals in $[-1,1]$ is called a chain if $G_{i}$ is the maximal interval such that $f\left(G_{i}\right) \subseteq G_{i+1}, i=0, \ldots, l-1$. We shall also call $G_{0}$ a pullback of $G_{l}$ along $\left\{x, \ldots, f^{l}(x)\right\}$ for $x \in G_{0}$.

We notice that for any chain $\left\{G_{i}\right\}_{i=0}^{l}$, the boundary of $G_{i}$ is mapped by $f$ onto the boundary of $G_{i+1}$. The multiplicity of intersection of a chain is the maximum number of intervals from it that has non-empty intersection.

Let $T \supset J$ be intervals and $L, R$ the components of $T \backslash J$, and define $C(T, J)=$ $(|T||J|) /(|L||R|)$ to be their cross-ratio. Let $T^{\prime} \supset J^{\prime}$ be components of $f^{-1}(T)$ and $f^{-1}(J)$. Then if $f$ is $C^{2}$ (in fact $C^{1+Z y g m u n d}$ is enough) and has non-flat critical points (in the sense defined in the introduction of this paper), then there exists $\gamma(f)>0$ (which does not depend on $T \supset J$ ) such that

$$
\frac{C(T, J)}{C\left(T^{\prime}, J^{\prime}\right)} \geq \begin{cases}1-\gamma(f)|T| & \text { if } T^{\prime} \text { does not contain a critical point } \\ \gamma(f) & \text { if } T^{\prime} \text { does contain a critical point }\end{cases}
$$

and

$$
|D f(x)| \leq \frac{1}{\gamma(f)} \frac{\left|f\left(T^{\prime}\right)\right|}{\left|T^{\prime}\right|} \text { for each } x \in T^{\prime}
$$

see [17]. It will be convenient to assume that if $M$ is an interval, $f$ is also defined on a $1 / \gamma(f)$-scaled neighbourhood $M^{\prime}$ of $M$ and that the above inequalities even hold if we take $J \subset M$ and $T \subset M^{\prime}$. 
From equations (11), (2) we get (see [17])

Lemma 1 (Koebe distortion). Let $\left\{G_{i}\right\}_{i=0}^{l}$ and $\left\{H_{i}\right\}_{i=0}^{l}$ be chains such that $G_{0} \supset$ $H_{0}$ and such that $G_{l}$ is a $\sigma$-scaled neighbourhood of $H_{l}$, for some $\sigma>0$. If the multiplicity of intersection of $\left\{G_{i}\right\}_{i=0}^{l}$ is bounded by $\kappa$, then the following hold.

(1) $G_{0}$ is an $\alpha$-scaled neighbourhood of $H_{0}$, where $\alpha>0$ depends only on $\sigma, \kappa$ and the constant $\gamma(f)$ from equation (11).

(2) If $G_{i_{1}}, \ldots, G_{i_{\nu}}$ are the intervals of the chain $\left\{G_{i}\right\}_{i=0}^{l}$ that contain critical points with $i_{1}<i_{2}<\cdots<i_{\nu}<i_{\nu+1}=l$, then the maps

$$
\left.f^{i_{j+1}-i_{j}-1}\right|_{G_{i_{j}+1}}: G_{i_{j}+1} \rightarrow G_{i_{j+1}}, \quad \text { for any } j=1, \ldots, \nu
$$

satisfy

$$
\frac{\left|D f^{i_{j+1}-i_{j}-1}(x)\right|}{\left|D f^{i_{j+1}-i_{j}-1}(y)\right|} \leq K
$$

for any $x, y \in H_{i_{j}+1}$, where $K<\infty$ depends only on $\sigma, \kappa$ and the constant $\gamma(f)$ from equation (1).

(3) $\left|D f^{l}(x)\right| \leq K^{\prime} \frac{\left|H_{l}\right|}{\left|H_{0}\right|}$ for all $x \in H_{0}$

where $K<\infty$ depends only on $\sigma, \kappa$ and the constant $\gamma(f)$ from equations (11) and (2).

Proof. For the first two statements, see 17. The third assertion holds by the Chain Rule, the second assertion and because of (2) applied to $T=H_{i_{j}}, j=1, \ldots, \nu$.

One of the main tools for obtaining Koebe space (which we need, together with Lemma 3, in order to apply the previous lemma) is the following analogue of a fact that is well known if the map is unimodal, non-renormalizable and has negative Schwarzian derivative.

Lemma 2 (Existence of Koebe space). There exists $\rho_{0}$ that only depends on the constant $\gamma(f)$ defined in equations (11) and (2) with the following properties. Let $I \subset M$ be a nice interval and $J_{1}, J_{2}$ return domains (not necessarily different) to $I$ with return times $r_{1}, r_{2}$, respectively. Then there exists an interval $\mathcal{M}_{i} \supset I$ that is a $\rho_{0}$-scaled neighbourhood of either $J_{1}$ or $J_{2}$ and that contains at most $2^{b+1}+2$ of the intervals $f^{j}\left(J_{l}\right), j=1, \ldots, r_{l}-1$ and $l=1,2$. Here $b$ is the number of turning points of $f$.

Note that the assertion of the lemma does not exclude the possibility that the $\mathcal{M}_{i}$ intersect (but do not contain) one or two of those intervals. If $M$ is an interval, and $I$ is very close to a boundary of $M$, then it is possible that $\mathcal{M}_{i}$ contains a neighbourhood (in the real line) of a boundary point of $M$ (this is the reason why we assumed that $f$ is defined on a $1 / \gamma(f)$-scaled neighbourhood of $M)$. This will not cause any difficulties, because we will only consider pullbacks of $\mathcal{M}_{i}$. It is quite easy to prove a similar lemma for the case where we consider more than two components of the return map to $I$.

Proof. Define the collections $\mathcal{B}_{i}=\left\{f\left(J_{i}\right), \ldots, f^{r_{i}}\left(J_{i}\right)\right\}$ and $\mathcal{B}=\mathcal{B}_{1} \cup \mathcal{B}_{2}$. Let $I_{l, i}$ be the interval containing $f^{l}\left(J_{i}\right)$ in the pullback of $I$ along $\left\{f^{l}\left(J_{i}\right), \ldots, f^{r_{i}}\left(J_{i}\right)\right\}$ and define the collections $\mathcal{C}_{i}=\left\{I_{l, i} ; l=1, \ldots, r_{i}\right\}$ and $\mathcal{C}=\mathcal{C}_{1} \cup \mathcal{C}_{2}$.

Let us define integers $m_{i}, 1 \leq i \leq 2$ and an interval $M_{m_{1}} \supset f^{m_{1}}\left(J_{i}\right)$ which we will pull backwards to get the scaled neighbourhood $\mathcal{M}_{i}$ of the statement. First note that two intervals from the collection $\mathcal{C}$ defined above are either disjoint or 
coincide. Let $f^{m_{1}}\left(J_{i}\right)$ be the smallest (shortest) interval from the collection $\mathcal{B}$. Then define $M_{m_{1}}:=L \cup I_{m_{1}, i} \cup R$, where $L, R$ are intervals adjacent to $I_{m_{1}, i}$ with $|L|=|R|=\rho_{0}^{\prime}\left|f^{m_{1}}\left(J_{i}\right)\right|$. Here we can take $\rho_{0}^{\prime}=1$ if there are intervals from $\mathcal{B}$ on both sides of $I_{m_{1}, i}$, but otherwise we can take $\rho_{0}^{\prime}=1 / \gamma(f)<1$ where $\gamma(f)$ is such that equations (1) and (2) hold for intervals inside a $1 / \gamma(f)$-scaled neighbourhood of $M$.

Note that $M_{m_{1}} \supset I_{m_{1}, i}$ is a $\rho_{0}^{\prime}$-scaled neighbourhood of $f^{m_{1}}\left(J_{i}\right)$, and it contains no other interval from $\mathcal{B}_{i}$. Now consider the chain $\left\{M_{t}\right\}_{t=0}^{m_{1}}$ such that $M_{t} \supset f^{t}\left(J_{i}\right)$.

If the map $f_{\mid M_{0}}^{m_{1}}: M_{0} \rightarrow M_{m_{1}}$ is surjective, then, for $0 \leq t \leq m_{1}, M_{t}$ does not contain any interval from $\mathcal{C}_{i}$ except $I_{t, i}$ (it may contain another interval from $\mathcal{C}_{j}$, $j \neq i)$. So in this case it follows that the chain $\left\{M_{t}\right\}_{t=0}^{m_{1}}$ has intersection multiplicity $\leq 3$. Applying Lemma 1 we get a constant $\rho_{0}=\rho_{0}\left(\rho_{0}^{\prime}\right)$ so that $M_{0}$ is a $\rho_{0}$-scaled neighbourhood of $J_{i}$ and so define $\mathcal{M}_{i}:=M_{0}$. This proves the lemma in this case.

However, even if the map $f_{\mid M_{0}}^{m_{1}}: M_{0} \rightarrow M_{m_{1}}$ is not surjective, if $0 \leq s, t \leq m_{1}$ with $s \neq t$ and $M_{s} \supset I_{t, i}$, then $s<t$. Indeed, otherwise $M_{m_{1}}$ would contain at least 2 intervals from $\mathcal{B}_{i}$, which is not true.

Let us first deal with the case that $J_{1}=J_{2}=I$ is a periodic interval of period $r=r_{1}=r_{2}$. By the previous remark if $0 \leq s, t \leq m_{1}$ with $s \neq t$ and $M_{s} \supset I_{t, i}$, then $s<t$. We claim that in this case $M_{m_{1}}$ intersects $I_{t^{\prime}, i}$ where $t^{\prime}=t+m_{1}-s(\bmod r)$. Indeed, if $f^{\tilde{s}}\left(I_{t, i}\right) \subset I$ for some $0 \leq \tilde{s}<m_{1}-s$, then $f^{\tilde{s}+1}\left(M_{s}\right)$ intersects $f(I) \subset I_{1}$, and so the claim follows. Hence, each interval $M_{t}, t=0,1, \ldots, m_{1}$, contains at most 3 intervals from $\mathcal{C}$. In particular, this chain has intersection multiplicity $\leq 8$ in this case.

Next consider the case that $I$ is not contained in a maximal periodic interval that is strictly contained in $M$. Consider those intervals $M_{n_{1}}, \ldots, M_{n_{\zeta}}$ from the collection $\left\{M_{t}\right\}_{t=0}^{m_{1}}$ that are mapped by $f$ strictly inside $M_{n_{j}+1}$. Hence, for each $i=1, \ldots, \zeta, f\left(\partial M_{n_{i}}\right)$ consists of one point and $M_{n_{i}}$ contains a turning point $c_{i}$ of $f$ such that the interval $f\left(M_{n_{i}}\right)$ is bounded by $f\left(c_{i}\right)$ and $f\left(\partial M_{n_{i}}\right)$. Assume that $M_{n_{j}} \cap M_{n_{k}} \neq \emptyset$, for some $n_{1} \leq n_{j}<n_{k} \leq n_{\zeta}$. Then $I_{n_{j}, i}$ is not contained in $M_{n_{k}}$. Hence, if $M_{n_{j}}$ contains the turning point $c_{k} \in M_{n_{k}}$ mentioned above, then because these intervals are pullbacks, $M_{n_{j}} \supset M_{n_{k}}$, which contradicts the assumption made in this paragraph. So $M_{n_{k}}$ contains a turning point of $f$ that is not contained in $M_{n_{j}}$. Moreover, the boundary of $M_{n_{j}}$ is mapped by $f$ inside $f\left(M_{n_{k}}\right)$. Now take $M_{n_{j}}$ maximal, in the sense that $M_{n_{j}} \cap M_{n_{k}} \supset M_{n_{i}} \cap M_{n_{k}}$, for each $n_{i}<n_{k}$. We claim that $M_{n_{l}} \cap M_{n_{j}} \cap M_{n_{k}} \neq \emptyset$ does not happen for $n_{l}, n_{j}<n_{k}, \quad n_{l} \neq n_{j}$. Indeed, since $f\left(\partial M_{n_{j}}\right) \in f\left(M_{n_{k}}\right)$, otherwise $f\left(M_{n_{l}}\right) \subset f\left(M_{n_{j}}\right)$, it follows that $M_{n_{l}} \subset M_{n_{j}}$. But again this contradicts the assumption made in this section. It follows that the multiplicity of intersection of the collection $\mathcal{D}:=\left\{M_{n_{1}}, \ldots, M_{n_{\zeta}}\right\}$ is at most equal to 2 . In particular, $\zeta \leq 2 b$.

We claim that $M_{n_{\zeta-j}}$ contains at most $j+4$ intervals from $\mathcal{C}$. Indeed, first observe that $M_{n_{\zeta}}$ contains at most 4 intervals from $\mathcal{C}$; otherwise it would contain at least 5 intervals, and therefore $M_{m_{1}}$ would contain at least 2 intervals from $\mathcal{C}$, contradicting the definition of $M_{m_{1}}$. Let us proceed by induction on $j$ and assume that $M_{n_{\zeta-(j-1)}}$ contains at most $j+3$ intervals from $\mathcal{C}$. Then, if $M_{n_{\zeta-j}}$ contains at least $j+5$ intervals from $\mathcal{C}$, the interval $M_{n_{\zeta-(j-1)}}$ would contain at least $j+4$ intervals from $\mathcal{C}$, a contradiction again.

This also implies that for $n_{\zeta-j-1}<t<n_{\zeta-j}$, the interval $M_{t}$ contains at most $j+4$ intervals from $\mathcal{C}$. Since $\zeta \leq 2 b$, we conclude that any interval $M_{t}$ contains at 
most $2 b+3$ intervals from $\mathcal{C}$. Next we claim that the multiplicity of intersection of $\left\{M_{t}\right\}_{t=0}^{m_{1}}$ is bounded by $4 b+7$. Indeed, notice that $M_{t}$ contains $I_{t, i}$ and that the intervals $I_{1, i}, \ldots, I_{m_{1}, i}$ are pairwise disjoint. So if $M_{t_{1}}, \ldots, M_{t_{4 b+8}}$, have a point in common, then one of these, say $M_{t_{s}}$, must contain at least $2 b+4$ intervals from $\mathcal{C}$, which again gives a contradiction.

Finally, we need to deal with the case that $I$ might be contained in a (maximal) periodic interval that is strictly contained in $M$. If the length of a nested chain of intervals from $\mathcal{D}$ is at most 2 , we still can argue as before and get that $\zeta \leq 4 b$. The multiplicity of intersection of $\left\{M_{t}\right\}_{t=0}^{m_{1}}$ in this case is at most $8 b+7$. If the length of a nested chain of intervals from $\mathcal{D}$ is at least 3 , then $I$ is contained in an $l$-periodic interval that contains at least 3 intervals from $\mathcal{C}$. Let $l$ be maximal such that $I$ is contained in a periodic interval with period $l$, and let $T$ be the corresponding maximal periodic interval containing $I$. Applying the proof of this lemma for the periodic case together with Lemma 3 to $f^{l} \mid T$, we get that the derivative of $f^{l}$ on $T$ is bounded by some universal number $N$. Let $I_{i}$ be the shortest interval from $\mathcal{C}$ in $T$. If there are intervals from $\mathcal{C}$ in both components of $T \backslash I_{i}$, then take $m_{i}=i$ and $M_{m_{1}}$ to be a 1-scaled neighbourhood of $I_{m_{1}}$. If $I_{i}$ has only elements from $\mathcal{C}$ in one component of $T \backslash I_{i}$, then (since there are at least 3 intervals from $\mathcal{C}$ in $T$ ) consider the pullback of $I_{i}$ by $f^{-l}$ or $f^{-2 l}$ and thus obtain an interval $I_{m_{1}}$ from $\mathcal{C}$ in $T$ and a $1 / M^{2}$-scaled neighbourhood of $I_{m_{1}}$ inside $T$ that does not contain any interval from the collection $\mathcal{C}$. So now we can apply what we did before to $f_{\mid T}^{l}$. Since this map has at most $2^{b}-1$ turning points, we conclude that any interval $M_{t}$ contains at most $2^{b+1}+2$ intervals from $\mathcal{C}$ and the multiplicity of intersection of $\left\{M_{t}\right\}_{t=0}^{m_{1}}$ is at most $2^{b+2}+5$. Since $M_{m_{1}}$ is a scaled neighbourhood of $f^{m_{1}}\left(J_{i}\right)$, it follows from Lemma 1 that $\mathcal{M}:=M_{0}$ is a scaled neighbourhood of $J_{i}$, which satisfies our statement.

The previous lemma implies that pullbacks of $\mathcal{M}_{i}$ along $J_{j}, \ldots, f^{r_{j}}\left(J_{j}\right)$ have bounded intersection multiplicity:

Lemma 3. Let $J$ be a return domain of a nice interval $I$ with return time $r$. Assume that $T \supset I$ contains at most $e$ of the intervals $I_{1}, \ldots, I_{r}=I$, where $I_{j}$ are the pullbacks of $I$ with $I_{j} \supset f^{j}(J)$. Then the following holds. Let $\left\{T_{i}\right\}_{i=0}^{r}$ be the chain such that $T_{r}=T$ and $T_{i} \supset f^{i}(J)$. Then the multiplicity of intersection of this chain is bounded by $2(e+b(e+2))+1$. In particular, if $T_{n_{1}}, \ldots, T_{n_{\nu}}$ are the intervals from $\left\{T_{i}\right\}_{i=0}^{r}$ that contain critical points, then $\nu \leq 2(e+b(e+2))+1$.

Proof. As in the previous lemma we can assume that $f$ is non-renormalizable (or more precisely, that the smallest periodic interval containing $I$ is equal to $M)$. First we prove that at most $b(e+2)$ of the intervals $T_{1}, \ldots, T_{r}$ contain turning points. Indeed, since $f$ is non-renormalizable, if for $0 \leq i_{1}<\ldots<i_{j} \leq r$ the intervals $T_{i_{1}}, \ldots, T_{i_{j}}$ contain the same turning point, then $T_{i_{1}} \subset T_{i_{2}} \subset \ldots \subset T_{i_{j}}$. Therefore $T_{r}$ contains the intervals $f^{r+i_{1}-i_{j}}(J), f^{r+i_{2}-i_{j}}(J), \ldots, f^{r}(J)$ and so $j \leq e+2$. Since $f$ has $b$ turning points we conclude that at most $b(e+2)$ of the intervals $T_{1}, \ldots, T_{r}$ contain turning points. This implies that an interval $T_{i}$ contains at most $e+b(e+2)$ of the intervals $I_{1}, \ldots, I_{r}=I$. Indeed, if for example $T_{i} \supset I_{m}$ and $I_{m}$ contains a turning point, then $T_{i+1}$ might not contain $I_{m+1}$. So each time an iterate of $T_{i}$ meets a turning point, one interval could be 'lost'. So in total one can 'lose' $b(e+2)$ intervals. Hence, the intersection multiplicity of $\left\{T_{i}\right\}_{i=0}^{r}$ is bounded by $2(e+b(e+2))+1$. 
By applying Lemma 2 to the case that $J=J_{1}=J_{2}$, using the disjointness which is provided by Lemma 3 for the pullback of $\mathcal{M}_{i}$, we immediately get the following distortion result from Lemma 1.

Corollary 1. Let I be a nice interval with return domain $J$, and denote the first return map by $\phi: J \rightarrow I$. If $|J| /|I|$ is close to 1 , define $K=J$. Otherwise define $K=\phi_{\mid J}^{-1}(J)$. Then the restriction of $\phi$ to a definite neighbourhood of $K$ is quasipolynomial: it can be written as a composition of at most $d$ maps of the form $L_{1} \circ f \circ L_{2}$ where $L_{1}, L_{2}$ have bounded distortion, $f$ is the original map and $d$ is the number of critical points.

\section{First RETURN MAPS}

In this section we are going to prove some important metric properties of individual branches of a first return map. It will be fundamental later on, when we will be pulling back Koebe space through many different branches. So, here we fix a nice interval $I$ with its first return map, and we will be in general analyzing the action of $\phi$ in one return domain $J \subset I$.

Definition 4. Let $J \subset I$ be a return domain, and let $x \in J$ and $m>0$ be such that $x, \ldots, \phi^{m-1}(x) \in J$ and $\phi^{m}(x) \notin J$. Then the fundamental domain of $\phi$ containing $x$ is the maximal interval $D \ni x$ for which $D, \phi(D), \ldots, \phi^{m-1}(D) \subset J$ and $\phi^{m}(D) \subset I \backslash J$.

From this definition it follows that the intervals $D, \ldots, \phi^{m}(D)$ are pairwise disjoint. If $\phi_{\mid J}$ is monotone, then $\phi^{m}(D)$ is equal to a connected component of $I \backslash J$ and the above definition of fundamental domain coincides with the usual one.

Definition 5. Take $\alpha>0$ and let $A$ be an interval in $I$ and $H$ a connected component of $I \backslash A$. If $|H|<\alpha|A|$, then we call $H$ an $\alpha$-small side of $A$ in $I$; otherwise we call $H$ an $\alpha$-big side of $A$ in $I$ (often we omit the reference to $I$ if it is clear).

Since we shall repeatedly introduce constants, we shall say that a constant $\alpha$ is universal if it only depends on previous constants. Often we will omit constants that are universal; for example, if $V$ is an $\alpha$-scaled neighbourhood of $U$ with $\alpha>0$ universal, then we say that $V$ is a scaled neighbourhood of $U$ or also that $U$ is wellinside $V$. The same convention will be used for $\alpha$-big sides and other analogous situations.

Take a domain $J$ on which $\phi$ is non-monotone. Notice that if $\phi_{\mid J}$ is a composition of maps with at most one turning point, then $\phi_{\mid J}$ is special: $\phi(\partial J)$ consists of just one point in $\partial I$. Moreover, $\phi(J)$ is equal to $\phi\left(J \backslash J^{\prime}\right)$ where $J^{\prime} \subset J$ is an interval and $J \backslash J^{\prime}$ consists of two intervals on which $\phi$ is monotone (whose endpoints are turning points of $\phi_{\mid J}$ and boundary points of $J$ ). In any case, if we denote by $J^{1}, \ldots, J^{l}$ the domains of monotonicity of $\phi: J \rightarrow I$ labelled from left to right, then $J^{1}, J^{l}$ are called the external branches of $J$, or the external intervals of monotonicity of $\phi_{\mid J}$. When $\phi: J \rightarrow I$ is surjective, then it is called orientation preserving (resp. reversing) if it is so at least on both external branches (i.e., on $J$ if $\phi$ is monotone). So we do not need that $\phi$ is monotone for it to be orientation preserving. If $\phi: J \rightarrow I$ is not surjective, then this notion is simply not defined.

Lemma 4. There exists $\delta_{1}>0$ such that for any return domain $J \subset I$ and any fundamental domain $D$ in $J$ that is not contained in the immediate basin of a 
periodic attractor, $D$ has at least one $\delta_{1}$-big side and $\phi(D)$ is contained in a $\delta_{1}$-big side of $D$.

Proof. Let us prove this lemma by contradiction: assume that $\phi(D)$ is contained in an $\alpha$-small side of $D$, with $\alpha>0$ small. Then $J$ has an $\alpha$-small side, and $\phi(D)$ is contained well-inside the interval $\mathcal{M}$ given by Lemma 2 (applied to the collection consisting of just $J$ ). In particular, the pullback of a definite neighbourhood of $\phi(D)$ is a definite neighbourhood $V$ of $D$. But if $\alpha$ is small, this implies that $\phi(V) \subset V$ and (by the last property of Lemma 1 and Lemma 3), that $\left|\phi^{\prime}(x)\right|<1$ for each $x \in V$. Hence $D$ is contained in the immediate basin of a periodic attractor of $f$, a contradiction.

Fix $\delta_{1}$ as in the previous lemma.

Lemma 5. For each $\xi>0$ sufficiently small, there exists $\delta_{2}>0$ with the following property. Let $J$ be a return domain of $\phi$, let $D$ be a fundamental domain in $J$ and $k \geq 1$ be so that $D, \ldots, \phi^{k-1}(D) \subset J$ while $\phi^{k}(D) \cap J=\emptyset$. If $D$ is not $\delta_{2}$-well-inside $I$, then the following hold

- $J$ has at least one $\xi$-small side;

- there exists a maximal external interval of monotonicity $J^{e} \subset J$ of $\phi_{\mid J}$ that is adjacent to a $\xi$-small side $H$ of $J$ and such that $D, \ldots, \phi^{k-2}(D) \subset J^{e}$, and $\phi^{k}(D) \cap H=\emptyset$;

- the intervals $D, \ldots, \phi^{k}(D)$ lie ordered, each of the intervals $D, \ldots, \phi^{k-1}(D)$ has a $\xi$-small side; (by the previous lemma $\phi^{i+1}(D)$ lies in the $\delta_{1}$-big side of $\phi^{i}(D)$ for $\left.i=0, \ldots, k-1\right)$;

- if $k \geq 2$, then $\phi_{\mid J^{e}}$ is orientation preserving;

- if both sides of $J$ are $\xi$-small, then $D \subset J^{e}, \phi_{\mid J}$ is orientation reversing and $k=1$.

Proof. Assume $D$ is not well-inside $I$. Then $J$ has at least one small side. Let $\mathcal{M}$ be the interval given by Lemma 2 associated to the collection $\{J\}$. Using the properties of this interval $\mathcal{M}$ it follows that all intervals of monotonicity of $\phi_{\mid J}$ restricted to $\phi_{\mid J}^{-1}(J)$, except possibly the external ones, are well-inside $I$. If both sides of $J$ are small, then all non-external branches of $\phi_{\mid J}$ are well-inside $I$. Moreover, $\phi_{\mid J}^{-1}(J)$ is well-inside $J$, whenever $\phi_{\mid J}$ is not monotone and $\phi_{\mid J}(\partial J) \in \partial I$ is contained in the closure of a big side of $J$.

Let us first consider the case that one side of $J$ is big. If $k=1$, then it follows from the previous lemma that $\phi(D)$ is contained in the big side of $J$, completing the proof of the lemma in this case. So let us assume that $k \geq 2$. Then the boundary point of $J$ on the small side of $J$ is mapped to the small side of $J$, because otherwise $\phi_{\mid J}^{-1}(J)$ and therefore $D$ is well-inside $I$. So we may assume (if $k \geq 2$ ) that $D$ intersects the interval $J^{e}$ of monotonicity of $\phi_{\mid J}$ that is adjacent to a small side of $J$ and that $\phi$ is orientation preserving on this interval $J^{e}$. If $\phi_{\mid J^{e}}^{\prime}$ is universally bounded, then it follows that $D$ must be well-inside $I$, a contradiction. If this derivative is not universally bounded, then by Lemma 3 it follows that $\phi\left(J^{e}\right) \supset J$ (and hence $\phi_{\mid J^{e}}$ has a fixed point). Since $k \geq 2$, this implies that $D$ is contained in $J^{e}$. Since each component of $\phi_{\mid J}^{-1}(J)$ except the one contained in $J^{e}$ is well-inside $I, D, \ldots, \phi^{k-2}(D) \subset J^{e}$. By the previous lemma, for $i=0, \ldots, k-1, \phi^{i}(D)$ has at least one $\delta_{1}$-big side that contains $\phi^{i+1}(D)$. It is sufficient to prove that for $i>0$ 
at least one side of $\phi^{i}(D)$ is small and that the other is $\gg 1$ big. Let $U$ be the interval connecting $D$ with the fixed point $p$ of $\phi_{\mid J^{e}}$. Since $D$ is not well-inside $I$, $D$ lies on the side $J^{e} \backslash\{p\}$ that is not adjacent to the small side of $J$ and, moreover, $\phi(U)$ is large compared to $U$. Hence, from part (3) of Lemma 1 and Lemma 3 it follows that $\phi^{i+1}(U)$ is also large compared to $\phi^{i}(U)$. This shows that one side of $\phi^{i}(D)$ is small, and so the proof is completed in this case.

If both sides of $J$ are small, then $\mathcal{M}$ contains a definite neighbourhood of $I$ and $\phi_{\mid J}^{\prime}$ is universally bounded. So adjacent fundamental domains associated to periodic points of $\phi_{\mid J}$ of period $\leq 2$ are of comparable size. From this it follows that $D$ is wellinside $I$, except possibly if $\phi(D) \cap J=\emptyset$ (i.e. $k=1$ ) and $D$ intersects an external domain $J^{e}$ of monotonicity of $\phi_{\mid J}$ such that $\phi_{\mid J^{e}}$ is orientation reversing.

Lemma 6. For each $\rho>0$ sufficiently small, there exists $\delta_{3}>0$ such that if $I$ is a $\rho$-scaled neighbourhood of an interval $V \subset I \backslash J$, then $J$ is a $\delta_{3}$-scaled neighbourhood of any component $A$ of $\phi_{\mid J}^{-k}(V)$ (where $k \geq 1$ is arbitrary).

Proof. Let $D$ be the fundamental domain containing $A$. If $\phi(D)$ is well-inside $I$, then $D$ is well-inside $J$. So if the assertion of this lemma does not hold, then $k$ is large and $\phi(D)$ is not well-inside $I$. From the previous lemma, it follows that the disjoint intervals $\phi(D), \ldots, \phi^{k-1}(D), \phi^{k}(D)$ lie ordered in $I$ and that $\phi^{i+1}(D)$ lies in the unique $\delta_{1}$-big side of $\phi^{i}(D)$ for $i<k$. Since $\phi^{k-1}(D)$ has a $\xi$-small side (which contains $\phi^{k-2}(D)$ ) and its $\delta_{1}$-big side contains $V$ and since $V$ is well-inside $I$, the interval $V$ is also well-inside the $\delta_{1}$-big side $W$ of $\phi^{k-2}(D)$. But then we can pull back $W$ with intersection multiplicity $\leq 2$ to a definite neighbourhood of $A$, and so we are done.

\section{VISITED DOMAINS AND JUMPING TIMES}

Let $I \subset M$ be a nice interval, and take a point $x \in I$ that visits $I$ infinitely many times. Let us associate to $x \in I$ the sequence of visited domains $\left\{J_{i}(x, I)\right\}_{i=0}^{\infty}$ and the sequence of jumping times $\left\{k_{i}(x, I)\right\}_{i=0}^{\infty}$ defined by taking $k_{0}(x, I):=0$ and inductively for all $i \geq 0$,

$$
\phi^{k_{i}(x, I)+j}(x) \in J_{i}(x, I), \quad 0 \leq j<k_{i+1}(x, I)-k_{i}(x, I)
$$

and

$$
\phi^{k_{i+1}(x, I)}(x) \notin J_{i}(x, I) .
$$

If $\phi^{k_{i}(x, I)+j}(x) \in J_{i}(x, I)$ for all $j \geq 0$, then we define $k_{i+1}(x, I)=\infty$. Note that $k_{i}(x, I)=\infty$ can happen, for example, when $x$ is eventually periodic or when $f$ is an infinitely renormalizable map and the forward orbit of $x$ hits an interval which is mapped into itself by the first return map to $I$. Note also that $k_{i+1}(x, I)-k_{i}(x, I)>$ 1 corresponds to a 'saddle-node cascade'. Unless necessary, we will denote the visited domains and the jumping times without the dependence on $x$ and $I$.

Lemma 7. Given a nice interval $I$ and a point $x \in I$ we consider its sequences of visited domains and jumping times, $\left\{J_{i}\right\}_{i=0}^{\infty}$ and $\left\{k_{i}\right\}_{i=0}^{\infty}$, respectively. Let us assume that $k_{i}<\infty$ for all $i \geq 0$. Then for any $\rho>0$ and any $n>0$ there exists $i_{0}$ with $0 \leq i_{0} \leq n$ called the stopping time such that the following hold.

(1) For $0 \leq i<i_{0}$, at least one side $H_{i}$ of $J_{i}$ is $\rho$-small and $J_{i+1}$ lies on the other side $G_{i}$ of $J_{i}$. So, only if both sides of $J_{i}$ are $\rho$-small, $J_{i+1}$ is allowed to lie in a $\rho$-small side of $J_{i}$. 
(2) For $0 \leq i<i_{0},\left(J_{i}, J_{i+1}\right) \cap\left(\bigcup_{k=0}^{i} J_{k}\right)=\emptyset$.

(3) One of the following properties holds:

- Property $P_{1}$ : both sides of $J_{i_{0}}$ are $\rho$-big sides and $i_{0}<n$.

- Property $P_{2}: J_{i_{0}}$ has precisely one $\rho$-small side, and $J_{i_{0}+1}$ is contained in that side and $i_{0}<n$.

- Property $P_{3}$ (and not $P_{1}$ or $\left.P_{2}\right):\left(J_{i_{0}}, J_{i_{0}+1}\right) \cap\left(\bigcup_{k=0}^{i_{0}} J_{k}\right) \neq \emptyset$ and $i_{0}<n$. In this case we define, for later use, $t$ to be the largest integer with $0 \leq t<i_{0}$ and $J_{t} \subset\left(J_{i_{0}}, J_{i_{0}+1}\right)$.

- Property $P_{4}: i_{0}=n$ and so $\left(J_{i}, J_{i+1}\right) \cap\left(\bigcup_{k=0}^{i} J_{k}\right)=\emptyset$ for all $0 \leq i<n$.

In Statement (1) it is not claimed that $G_{i}$ is a $\rho$-big side. It is useful to have a graphic image to explain what Statements (1) and (2) of this lemma mean: the intervals are visited in a spiral fashion spiraling 'towards the center'. For example, connect $J_{i}$ and $J_{i+1}$ by a semicircle in the upper half plane. Statement (2) says that one can draw these semicircles without intersections. Property $P_{3}$ states that this spiral structure is broken and Property $P_{4}$ that the spiral structure is maintained until the $n$-th visit. Because of the spiral structure of the intervals $J_{0}, \ldots, J_{i_{0}}$ (and maximality of $t$ ), if Property $P_{3}$ holds for $i_{0}$, then $H_{t} \cap H_{i_{0}}=\emptyset$ and $G_{t} \cap G_{i_{0}} \neq \emptyset$.

Proof of Lemma 7. The proof of this lemma is essentially the definition of properties $P_{i}$.

In the next lemmas we show that one can pull back space if the spiral structure is preserved as in $P_{4}$ (many visits to the same domain or a long cycle between two visited domains is allowed), and that one gets space inside $I$ anyway if $P_{1}, P_{2}$ or $P_{3}$ holds.

From now on fix $\rho>0$ smaller than $\rho_{0} / 2$ (where $\rho_{0}$ is given by Lemma 22), so it makes sense to say that one of the properties $P_{i}$ is satisfied.

\subsection{Pulling back space.}

Lemma 8. There exists $\sigma_{1}>0$ such that if $x \in I$ satisfies Property $P_{4}$ with $i_{0}=n$, and if all of the following properties are satisfied: (i) $J_{0}=J_{n}$ has precisely one small side, (ii) $\phi\left(J_{0}\right)$ contains the small side of $J_{0}$ and (iii) $\phi_{\mid} J_{0}$ is not an orientation reversing surjective branch (in the sense defined above Lemma 4), then $I$ is a $\sigma_{1}$-scaled neighbourhood of the pullback $U$ of $J_{i_{0}}=J_{n}$ along $\left\{x, \ldots, \phi^{k_{n}}(x)\right\}$.

Proof. Let $i_{0}^{\prime}>0$ be minimal so that $J_{i_{0}^{\prime}}=J_{0}$. We claim that the pullback of $J_{i_{0}^{\prime}}$ along $\left\{x, \ldots, \phi^{k_{i_{0}^{\prime}}}(x)\right\}$ is well-inside $I$. So, in other words, we claim that it is enough to consider the case that $i_{0}^{\prime}=n$ and so $J_{0}, \ldots, J_{n-1}$ are pairwise disjoint.

So let us assume this and prove the lemma in this case. Since $P_{4}$ holds, we can assume that $J_{0}, J_{n-1}, \ldots, J_{1}$ are ordered in $I$ from left to right. Note that $U$ is contained in a fundamental domain $D_{0}$, which by Lemma 5 has space to its right inside $I$. Analogously, $\phi^{k_{1}}(U)$ is contained in a fundamental domain $D_{1} \subset J_{1}$, which has space to its left inside $I$. It remains to show that there is space to the left of $U$ inside $I$.

Let $\mathcal{M}$ be the interval from Lemma 2 associated to the collection $\left\{J_{0}, J_{n-1}\right\}$. There are two possibilities.

Case 1. $\mathcal{M}$ is a $\rho_{0}$-scaled neighbourhood of $J_{n-1}$. Then also $J_{1}$ is well-inside $\mathcal{M}$, because $J_{1}=J_{n-1}$ or $J_{1}$ is to the right of $J_{n-1}$. As we observed before, $D_{1} \subset J_{1}$ has space on its left and if $\phi_{\mid J_{0}}$ is an orientation preserving surjective branch, we 
can pull back this space to a space on the left of $U$ inside $J_{0}$ (see Lemma 6). If $\phi_{\mid J_{0}}$ has at least one turning point, because $\phi\left(J_{0}\right)$ contains a small side of $J_{0}$, it must contain the big side of $D_{1}$, and then we get that the pullback of $D_{1}$ to $x$ is well-inside $J_{0}$.

Case 2. $\mathcal{M}$ is a $\rho_{0}$-scaled neighbourhood of $J_{0}$. Let us consider the fundamental domain $D_{n-1} \subset J_{n-1}$ that contains $\phi^{k_{n-1}}(U)$, and let $\tilde{\mathcal{M}}$ be the pullback of $\mathcal{M}$ by the extension of $\phi_{\mid J_{n-1}}$. By the same reason as above, $D_{n-1}$ has space on its left. Then $V=\phi_{\mid J_{n-1}}^{-1}\left(J_{n}\right) \subset D_{n-1}$ has space on its left and it is well-inside $\tilde{\mathcal{M}}$. Now if $V$ is well-inside $I$, using Lemma 6] we can pull it back to $\phi^{k_{n-1}}(x)$, and using the disjointness of $J_{0}, \ldots, J_{n-2}$ we get that $U$ is well-inside $J_{0} \subset I$. If $V$ is not well-inside $I$ and $n>2$, we have that $J_{1}$ is well-inside $\tilde{\mathcal{M}}$ and has space to its left. The same reasoning as in Case 1 can be applied. If $V$ is not well-inside $I$ and $n=2$, we also can apply the same reasoning as in Case 1 to pull back to $U$ the space on the left of $V$ (or, of $D_{1}$ in the case that $k_{2}-k_{1}>1$ ).

Lemma 9. For each $\xi>0$ there exists $\xi^{\prime}>0$ with the following property. Let $n>0$, and let $\left\{J_{i}\right\}_{i=0}^{n}$ and $\left\{k_{i}\right\}_{i=0}^{n}$ be, respectively, the first $n$ visited domains and jumping times of some point $x \in I$. Assume that property $P_{4}$ holds for $n$. Let $V \subset I$ with $\phi^{k_{n+1}}(x) \in V$ and such that $V$ is $\xi$-well-inside $I$. Then the pullback $V_{0}$ of $V$ along $\left\{x, \ldots, \phi^{k_{n+1}}(x)\right\}$ is $\xi^{\prime}$-well-inside $J_{0}$.

Proof. According to Lemma 6 the pullback $V_{n}$ of $V$ along $\left\{\phi^{k_{n}}(x), \ldots, \phi^{k_{n+1}}(x)\right\}$ is well-inside $J_{n}$. Since $P_{4}$ holds for $n$, there exists $i_{1} \leq n$ such that $\phi^{i_{1}}(x)$ is the first visit of $x$ to $J_{i_{1}}$ and such that

if $n-i_{1}=0 \bmod 2$, then $J_{i_{1}}=J_{i_{1}+2}=\cdots=J_{n}$ and $J_{i_{1}+1}=J_{i_{1}+3}=\cdots=J_{n-1}$

and

if $n-i_{1}=1 \bmod 2$, then $J_{i_{1}}=J_{i_{1}+2}=\cdots=J_{n-1}$ and $J_{i_{1}+1}=J_{i_{1}+3}=\cdots=J_{n}$.

It is enough to show that the pullback $V_{i_{1}}$ of $V_{n}$ along $\left\{\phi^{k_{i_{1}}}(x), \ldots, \phi^{k_{n}}(x)\right\}$ is well-inside $J_{i_{1}}$, because the further pullback along $\left\{x, \ldots, \phi^{k_{i_{1}}}(x)\right\}$ of $J_{i_{1}}$ is disjoint $\left(\phi^{k_{i_{1}}}(x)\right.$ is the first visit to $\left.J_{i_{1}}\right)$. If there exists an integer $i_{1} \leq i_{1}^{\prime} \leq n$ for which $\phi^{k_{i_{1}^{\prime}}}(x)$ is contained in a fundamental domain of $J_{i_{1}^{\prime}}$ that is $\delta_{2}$ well-inside $J_{i_{1}^{\prime}}$, then it is enough to pull back this space along $\left\{\phi^{k_{i_{1}}}(x), \ldots, \phi^{k_{i_{1}^{\prime}}}(x)\right\}$. This means that we may assume that $\phi^{k_{i_{1}}}(x), \ldots, \phi^{k_{i_{1}^{\prime}}}(x)$ are contained in fundamental domains of $J_{i_{1}}, \ldots, J_{i_{1}^{\prime}}$, respectively, that are not $\delta_{2}$ well-inside $J_{i_{1}}, \ldots, J_{i_{1}^{\prime}}$. This means that we can apply Lemma 5 throughout the remainder of this lemma. In particular $\phi^{k_{i_{1}}}(x), \ldots, \phi^{k_{i_{1}^{\prime}}}(x)$ are all contained in fundamental domains of $J_{i_{1}}, \ldots, J_{i_{1}^{\prime}}$ that intersect the maximal external interval of monotonicity. Because of Lemma 6 it is also enough to assume that $i_{1}^{\prime}-i_{1}$ is large. So let us assume for example that $i_{1}^{\prime}-i_{1} \geq 6$ (we take $i_{1}$ the largest possible with this property), so that $J_{i_{1}}=$ $J_{i_{1}+2}=\cdots$ and $J_{i_{1}+1}=J_{i_{1}+3}=\cdots$ (where $\cdots$ refers to intervals up to $J_{i_{1}^{\prime}}$ or $\left.J_{i_{1}^{\prime}-1}\right)$. Then $\phi^{k_{i_{1}}}(x), \phi^{k_{i_{1}+2}}(x), \ldots, \phi^{k_{i_{1}^{\prime}-3}}(x)$ are all in an orientation preserving external branch of the first return to $J_{i_{1}}$. We can assume that the pullback $V_{i_{1}^{\prime}-1}$ of $V_{n}$ along $\left\{\phi^{k_{i_{1}^{\prime}-1}}(x), \ldots, \phi^{k_{n}}(x)\right\}$ is well-inside $J_{i_{1}}$ and using Lemma 6 the lemma follows. 
Lemma 10. There exists $\sigma_{2}>0$ such that if $x \in I$ satisfies Property $P_{1}$ or $P_{2}$ for some $i_{0}<n$, then the pullback of $J_{i_{0}+1}$ along $\left\{x, \ldots, \phi^{k_{i_{0}+1}}(x)\right\}$ is $\sigma_{2}$-well-inside I. If $i_{0}>0$, this pullback is $\sigma_{2}$-well-inside $J_{0}$.

Proof. If $P_{2}$ holds, then Lemma 4 implies that $I$ is a $\delta_{2}$-scaled neighbourhood of the fundamental domain $D$ containing $\phi^{k_{i}}(x)$. Obviously the same happen if $P_{1}$ holds. In either case it follows from Lemma 9 that the pullback of $D$ along $\left\{x, \ldots, \phi^{k_{i_{0}}}(x)\right\}$ into $J_{0}$ is well-inside $I$. If $i_{0}>0$, we get it well-inside $J_{0}$.

Lemma 11. There exists $\sigma_{3}>0$ such that if $x \in I$ satisfies Property $P_{3}$ for some $i_{0}<n$, then $I$ is a $\sigma_{3}$-scaled neighbourhood of the pullback $U \subset J_{t}$ of $J_{i_{0}+1}$ along $\left\{\phi^{k_{t}}(x), \ldots, \phi^{k_{i_{0}+1}}(x)\right\}$.

Proof. In order to be definite assume that $J_{t}$ is to the left of $J_{i_{0}}$. Let $G_{i_{0}}$ be the left side of $J_{i_{0}}$ (it contains $J_{t}$ ), and let $H_{t}$ be the left side of $J_{t}$ (which is small and contains $\left.J_{i_{0}+1}\right)$. Consider the first return map $\psi$ to $G_{i_{0}}$, and let $D, D^{\prime}$ be the fundamental domains containing $x$ of $\phi$ and $\psi$, respectively. Then $D^{\prime} \subset D$ and $D^{\prime}, \ldots, \psi^{k_{t+1}-k_{t}-1}\left(D^{\prime}\right)$ are in $J_{t}$ and $\psi \circ \psi^{k_{t+1}-k_{t}-1}\left(D^{\prime}\right) \subset J_{t+1}$. By Lemma 5 if $D^{\prime}$ is not well-inside $G_{i_{0}}$, then $D^{\prime}, \ldots, \phi^{k_{t+1}-k_{t}}\left(D^{\prime}\right)$ lie ordered, and always lie on a big side of the previous interval. This implies that there is space to the left of $D^{\prime}$. So it remains to show that there is space to the right of $D^{\prime}$ in $I$. So we may assume that both sides of $J_{t}$ are small. But then it follows from the last part of Lemma 5 that $D$ has space to its right inside $I$. Combined this shows that $D^{\prime}$ is well-inside $I$.

The previous lemmas can be combined:

Proposition 1 (Part (1) of Theorem C). For each $\xi>0$ there exists $\xi^{\prime}>0$ with the following property. Let $V$ be an interval $\xi$-well-inside $I$ and $x \in I$ such that $\phi^{k}(x) \in V$, for some $k \geq 1$. Then the pullback of $V$ along $\left\{x, \ldots, \phi^{k}(x)\right\}$ is $\xi^{\prime}$-well-inside the return domain to I containing $x$.

Proof. If $P_{4}$ holds, then the lemma follows from Lemma 9 If $P_{1}, P_{2}$ or $P_{3}$ hold, then this follows from the previous Lemmas 10 and 11 .

4.2. Corollary: There exist no wandering intervals. Of course this corollary is not new (it was proved in different generalities by Guckenheimer, de Melo \& van Strien, Lyubich, Blokh \& Lyubich and Martens \& de Melo \& van Strien. Let us show that our methods provide a new (and we believe simpler) proof of this fact.

Proof of the Corollary. Let us introduce some notation. We call a finite partition $\mathcal{P}$ of $M$ nice if boundary points of partition elements are mapped into boundary points. (Such a partition is generated by preimages of a finite number of periodic orbits.) Denote by $\mathcal{P}(x)$ the element of the partition $\mathcal{P}$ containing $x$ (this is unique if $x$ is not on a boundary of partition elements). Furthermore, denote by $\mathcal{L}_{x}(I)$ the component of the domain of the first entry map to $I$ containing $x$.

Let $W$ be an interval such that all its forward iterates are disjoint and assume by contradiction that $W$ does not converge to a periodic orbit. Let $z$ be an accumulation point of $f^{n}(W)$, take a nice partition $\mathcal{P}_{0}$, and let $I_{0}=\mathcal{P}_{0}(z)$. (We can assume that $f$ has a nice partition. Indeed, if $f$ has no fixed points, then by modifying $f$ on the wandering interval $W$ we can construct a new map $\tilde{f} \in \mathcal{A}^{1+Z y g m u n d}$, with possibly more turning points, with a wandering interval and with at least one fixed point.) Note that infinitely many iterates of $W$ are contained in $I_{0}$ and that, since all iterates of $W$ are disjoint, every point in $W$ simultaneously visits the same 
return domains of $I_{0}$. We claim that there exists a constant $\rho>0$ so that the first iterate of $W$ entering $I_{0}$ is contained in a nice interval $I_{0}^{\prime}$ that is $\rho$-well-inside $I_{0}$. Here $I_{0}^{\prime}$ is a component of a nice partition $\mathcal{P}_{1}$ that refines $\mathcal{P}_{0}$.

Let us first show that this proves the corollary. From the claim it follows that there exists a sequence of refining nice partitions $\mathcal{P}_{0}, \mathcal{P}_{1}, \ldots$ such that for each $n \geq 0$, the first iterate of $W$ entering $I_{n}=\mathcal{P}_{n}(x)$ is contained in a component $I_{n}^{\prime}$ of $\mathcal{P}_{n+1}$ that is $\rho$-well-inside $I_{n}$. By pulling back this space (using Lemma 1) we get

$$
\mathcal{L}_{W}\left(I_{n}^{\prime}\right) \text { is } \rho^{\prime} \text {-well-inside } \mathcal{L}_{W}\left(I_{n}\right)
$$

Moreover, since $I_{n}^{\prime}$ contains the first entry of $W$ to $I_{n}$ and since both $I_{n}^{\prime}$ and $I_{n+1}$ are components of $\mathcal{P}_{n+1}$,

$$
\mathcal{L}_{W}\left(I_{n+1}\right) \subset \mathcal{L}_{W}\left(I_{n}^{\prime}\right)
$$

Combining this gives that for each $n, W \subset \mathcal{L}_{W}\left(I_{n}\right)$ is $n \rho^{\prime}$-well-inside $\mathcal{L}_{W}\left(I_{0}\right)$. This gives a contradiction with the compactness of the dynamical space $M$.

So let us prove the claim. Notice that as in Lemma 7 either one of the properties $P_{1}, P_{2}, P_{3}$ holds at some moment, or $P_{4}$ holds forever, or $W$ eventually only visits one return domain.

Case 1. $P_{1}, P_{2}, P_{3}$ hold. Then the previous two lemmas and Proposition 1 imply that we can take for $I_{1}$ a pullback of a domain of $I_{0}$.

Case 2. $P_{4}$ holds forever. If $W$ keeps spiralling towards the center of $I_{0}$ visiting infinitely many different return domains in $I_{0}$, then eventually it visits a domain that is well-inside $I_{0}$, and so again Claim 1 holds (using Proposition 11). So the final two possibilities are that (during visits to $I_{0}$ ) either $W$ eventually only visits one return domain $J$ or that $P_{4}$ holds forever and $W$ eventually only visits two domains $J, J^{\prime}$ in $I_{0}$ both having one large side. Let us consider both of these possibilities next.

Case 3. All iterates of $W$ (entering $I_{0}$ ) eventually visit only one return domain $J$ of the first return map to $I_{0}$. So $W$ eventually enters a periodic interval $J^{\prime} \subset J$ (i.e., $\phi\left(J^{\prime}\right) \subset J^{\prime}$ and $\left.\phi\left(\partial J^{\prime}\right) \subset \partial J^{\prime}\right)$. Let $Q$ be the set of fixed points of $\phi: J^{\prime} \rightarrow J^{\prime}$. From Lemma 2 each component of $J^{\prime} \backslash \phi^{-1}(Q)$ that contains a turning point of $\phi$ is well-inside $J^{\prime}$. This lemma also implies that $\left|\phi^{\prime}\right|$ is universally bounded on $J^{\prime}$ and so all fundamental domains associated to fixed points of $\phi$ are well-inside $J^{\prime}$. It follows that some iterate of $W$ is contained in a component of $J^{\prime} \backslash \phi^{-k}(Q)$ that is well-inside $J^{\prime} \backslash I_{0}$. By Proposition 1, the first iterate of $W$ is contained in an element of $\mathcal{P}_{1}$ where $\mathcal{P}_{1}$ is the refinement of $\mathcal{P}_{0}$ obtained by adding the periodic points associated to $Q$ and appropriate preimages by $f$.

Case 4. $W$ eventually only visits two return domains $J_{1}, J_{2}$ of $I_{0}$; then we argue as follows. If either $\left|J_{1}\right| /\left|I_{0}\right|$ or $\left|J_{2}\right| /\left|I_{0}\right|$ is close to 1 , then $\left|\phi^{\prime}\right|$ is bounded on $J_{1}$ or $J_{2}$, and so fundamental domains of $\phi: J_{1} \rightarrow I$ or of $\phi: J_{2} \rightarrow I$ are well-inside $I$, and we can complete the argument as before. So assume that both these domains do not occupy almost all of $I_{0}$. Then consider the first return map to $J^{\prime}=J_{1}$. If for this first return map, we are in Case 1 or Case 2, then we argue as before. If we are again in Case 3 , then consider the two domains $J_{1}^{\prime}, J_{2}^{\prime}$ in $J^{\prime}$. If either $\left|J_{1}^{\prime}\right| /\left|J^{\prime}\right|$ or $\left|J_{2}\right| /\left|J^{\prime}\right|$ is close to 1 , then we are done as before. But if this does not hold, then either $J_{1}^{\prime}$ or $J_{2}^{\prime}$ is well-inside $I_{0}$ (here we use that $\left|J_{1}\right| /\left|I_{0}\right|,\left|J_{2}\right| /\left|I_{0}\right|,\left|J_{1}^{\prime}\right| /\left|J^{\prime}\right|$ and $\left|J_{2}^{\prime}\right| /\left|J^{\prime}\right|$ are all away from one). This means that we can pull back this space as before.

This completes the proof of the claim and of the corollary. 


\section{TheOrEms A And A': ReAl Bounds AT ARBITRARY NON-PERIODIC POINTS}

Let $I$ be a nice interval and $x \in I$ be a recurrent point. Let us show that one has real bounds around $x$ (often one will take for $x$ a critical point). Write $I_{0}=I$, let $\phi_{1}$ be the first return map to $I_{0}$, and let $I_{1}$ be the domain of this map containing $x$. Inductively one gets in this way a sequence of intervals $I_{n}$ and first return maps $\phi_{n+1}$ to $I_{n}$ such that $I_{n+1}$ is the domain of $\phi_{n+1}$ containing $x$. We say that the first return map $\phi_{n+1}$ to $I_{n}$ is non-central w.r.t. $x$ (if clear we will omit the reference to $x)$ if $\phi_{n+1}(x) \notin I_{n+1}$. Of course either $x$ is contained in a periodic interval or otherwise there are infinitely many $n$ 's for which $\phi_{n+1}: I_{n+1} \rightarrow I_{n}$ are non-central. If $x$ is not recurrent, then $I_{n+1}$ might be empty, so the results below become trivial.

Lemma 12. Assume that $I_{n-1}$ is well-inside $I_{n-3}$. Then

(1) if $\phi_{n-1}: I_{n-1} \rightarrow I_{n-2}$ is non-central, then $I_{n}$ is well-inside $I_{n-1}$;

(2) if $I_{n}$ is $\xi$-well-inside $I_{n-1}$, then for each $j \geq 1$, there exists $\xi(j)>0$ such that $I_{n+j}$ is $\xi(j)$-well-inside $I_{n+j-1}$.

Proof. Let us first prove that (1) holds. Note that $\phi_{n-1}\left(I_{n}\right)$ is in a component of $I_{n-2} \backslash I_{n-1}$ because $\phi_{n-1}: I_{n-1} \rightarrow I_{n-2}$ is non-central and that $\phi_{n-2} \circ \phi_{n-1}\left(I_{n}\right)$ is an iterate of $I_{n}$ before it returns to $I_{n-1}$. Hence from Proposition 1 and the assumption that $I_{n-1}$ is well-inside $I_{n-3}$ it follows that $\phi_{n-1}\left(I_{n}\right)$ is well-inside $I_{n-2}$. But this implies that $I_{n}$ is well-inside $I_{n-1}$.

Let us next prove (2). If $\phi_{n}$ is non-central, then $\phi_{n}\left(I_{n+1}\right)$ is an iterate of $I_{n+1}$ before it returns to $I_{n}$, and so we can again apply Proposition 1 and that $I_{n}$ is wellinside $I_{n-1}$ to conclude that $\phi_{n}\left(I_{n+1}\right)$ is well-inside $I_{n-1}$. This implies that $I_{n+1}$ is well-inside $I_{n}$. If $\phi_{n}$ is central, we have that $\phi_{n}\left(I_{n+1}\right) \subset I_{n}$ and $\phi_{n}\left(I_{n}\right) \subset I_{n-1}$ and that $I_{n+1}$ is well-inside $I_{n}$ follows immediately.

Lemma 13. If $\phi_{n}: I_{n} \rightarrow I_{n-1}$ and $\phi_{n+1}: I_{n+1} \rightarrow I_{n}$ are non-central returns, then $I_{n+2}$ is well-inside $I_{n}$.

Proof. Take a point $x \in I_{n+1} \subset I_{n}$ such that $\phi_{n+1}(x) \notin I_{n+1}$ and $\phi_{n}(x) \notin I_{n}$. Let $k>1$ be the smallest integer such that $\phi_{n}^{k}(x) \in I_{n}$ and consider $i_{0}$ to be the stopping time of $x$ such that $k_{i_{0}} \leq k$.

First observe that, if there exists a fundamental domain $D$ that is well-inside $I_{n-1}$ such that $\phi_{n}^{k_{i}}(x) \in D$ for some $k_{i} \leq k$, then from Proposition 1 it follows that the pullback of $D$ to $x$ is an interval well-inside $I_{n-1}$. Because this interval contains $I_{n+1}$ we get that $I_{n+1}$ is well-inside $I_{n-1}$, and this implies the lemma. So we can assume that $P_{1}$ and $P_{2}$ do not hold. If Property $P_{3}$ holds, it follows from Lemma 11 that the pullback $U \subset J_{t}$ (remember that $t$ is the largest integer with $0 \leq t<i_{0}$ and $\left.J_{t} \subset\left(J_{i_{0}}, J_{i_{0}+1}\right)\right)$ of $J_{i_{0}+1}$ to $\phi_{n}^{k_{t}}(x)$ is well-inside $I_{n-1}$ and by the same reasoning as before, now playing with $U$ instead of $D$, we are done in the same way. If Property $P_{4}$ holds, we have that $k_{i_{0}}=k$. Then if $I_{n}$ satisfies the hypothesis of Lemma 8 it follows that the pullback $U \subset I_{n}$ of $I_{n}$ along $\left\{x, \phi_{n}(x), \ldots, \phi_{n}^{k}(x)\right\}$ is well-inside $I_{n-1}$, and the same reasoning as before applies again.

So we assume from now on that $k_{i_{0}}=k, J_{i_{0}}=I_{n}$, Property $P_{4}$ holds for $x$ but $I_{n}$ does not satisfy the hypothesis of Lemma 8. It means that $I_{n}$ has two small sides, or $\phi\left(I_{n}\right)$ does not contain a small side of $I_{n}$ or $\phi_{n \mid I_{n}}$ is an orientation reversing surjective branch. 
Then we set $y=\phi_{n}(x)$ and define the smallest $s>0$ such that $\phi_{n}^{s}(y) \in V$, where $V=\phi_{n \mid I_{n}}^{-1}\left(J_{0}(y)\right)$. If such a $t$ does not exist, we set $s=\infty$. From Lemma 7 we know that one of the Properties $P_{1}, P_{2}, P_{3}$ or $P_{4}$ holds for $y$ with $k_{i_{0}(y)} \leq s$. If $k_{i_{0}(y)} \leq s$ and one of the Properties $P_{1}, P_{2}$ or $P_{3}$ holds, it follows from one of the situations analysed above that the pullback of $J_{i_{0}(y)+1}$ to $y$ is well-inside $I_{n-1}$. Pulling back once more to $x$ we get an interval that contains $I_{n+2}$ and is well-inside $I_{n}$ and the statement follows in this case. Let us assume that $P_{4}$ holds and $k_{i_{0}(y)}=s$, which means that $J_{i_{0}(y)}=I_{n}$.

We still need to consider two cases: (i) The visited domain $J_{i_{0}(y)-1}$ lies between $I_{n}$ and $J_{0}(y)$. Then, because $\phi_{n}^{s}(y) \in V$, we have that $P_{3}$ holds for $z=\phi_{m}^{s-1}(y)$ (with $k_{i_{0}(z)}=2$ ). So we can use Lemma11 and get that $\phi_{n \mid J_{i_{0}(y)-1}}^{-1}(V)$ is well-inside $I_{n-1}$, and we are done again. (ii) The visited domain $J_{i_{0}(y)-1}$ is equal to $J_{0}(y)$, which means that there is no visited domain between $I_{n}$ and $J_{0}(y)$. We can assume that $J_{0}(y)$ does not satisfy the hypothesis of Lemma 8 ; otherwise, this lemma would imply that the pullback $W$ of $V$ to $y$ is well-inside $I_{n-1}$. Then the pullback of $W$ to $x$ would be well-inside $I_{n}$, which would imply that $I_{n+2}$ is well-inside $I_{n}$, and the statement would follow. So we can assume that both $I_{n}$ and $J_{0}(y)$ do not satisfy the hypothesis of Lemma 8 . But then we consider the first return map to $I_{n}$ whose domain $I_{n+1}$ (containing $x$ ) either satisfies the hypothesis of Lemma 8 . is well-inside $I_{n-1}$, or it has two small sides. In each of these situations we get the required statement.

Lemma 14. Let $\phi_{m-1}: I_{m-1} \rightarrow I_{m-2}$ be non-central, while for some $n>m$, $\phi_{k}: I_{k} \rightarrow I_{k-1}$ is central for $k=m, \ldots, n-1$ and $\phi_{n}: I_{n} \rightarrow I_{n-1}$ is non-central. Then $I_{n+1}$ is well-inside $I_{n}$.

Proof. By assumption $\phi_{m}=\phi_{m+1}=\cdots=\phi_{n}$. Of course it is enough to show that $\phi_{n}\left(I_{n+1}\right)$ is well-inside a component of $I_{n-1} \backslash I_{n}$. Let us prove this. Since $\phi_{m}=\phi_{m+1}=\cdots=\phi_{n}:=\phi$, one gets that a component of $I_{n-1} \backslash I_{n}$ is mapped by $\phi^{j}$ to a component of $I_{n-1-j} \backslash I_{n-j}$ for $j=0, \ldots, n-m$, and all these iterates are pairwise disjoint. So it is enough to show that $\phi^{n-m}\left(I_{n+1}\right)$ is well-inside a component of $I_{m-1} \backslash I_{m}$ (note that $\phi^{n-m}\left(I_{n+1}\right)$ is an iterate of $I_{n+1}$ before it has returned to $\left.I_{n}\right)$. Note that $\phi_{m-1}: I_{m-1} \rightarrow I_{m-2}$ and $\phi_{m}: I_{m} \rightarrow I_{m-1}$ are noncentral w.r.t. $\phi_{n}^{n-m}\left(I_{n+1}\right)$, and the previous lemma implies that the return domain to $I_{m}$ that contains $\phi_{n}^{n-m}\left(I_{n+1}\right)$ is well-inside $I_{m-1}$. It follows that $I_{n} \subset I_{m+1}$ is well-inside $I_{m-1}$. Hence by Proposition 1 we get that $I_{n+1}$ is well-inside $I_{n}$.

5.1. Proof of Theorem A. Let us assume now, for $m>p$, that $\phi_{p}: I_{p} \rightarrow I_{p-1}$ and $\phi_{m}: I_{m} \rightarrow I_{m-1}$ are non-central and prove the first statement. It follows from Lemma 13 or 14 that $I_{m+2}$ is well-inside $I_{m}$. Because of this, if for $n>m$, $\phi_{n}: I_{n} \rightarrow I_{n-1}$ is also non-central, Lemma 12 or 14 implies that $I_{n+1}$ is well-inside $I_{n}$, and the statement follows.

Let us assume that $I_{n+1}$ is well-inside $I_{n}$ and prove the second statement of the theorem. Let $J$ be a domain of the first return map $\phi_{n+2}$ to $I_{n+1}$. If $\phi_{n+1}(J) \subset$ $I_{n+1}$, then the first part of the second statement holds because $\phi_{n+2 \mid J}=\phi_{n+1 \mid J}$. If $I_{n+1} \cap \phi_{n+1}(J)=\emptyset$, then by Proposition 1 the first part of the second statement holds because $\phi_{n+1}(J)$ is well-inside a domain of $\phi_{n}$ because it is a pullback of $I_{n+1}$ by some iterate of $\phi_{n}$ and $I_{n+1}$ is well-inside $I_{n}$. To prove that $\xi^{\prime}$ tends to infinity as $\xi$ tends to infinity, one needs also to consider the situation that the pullback of $I$ along the orbit $\left\{x, \ldots, \phi_{n}(x)\right\}$ has high intersection multiplicity. 
5.2. Proof of Theorem $\mathbf{A}^{\prime}$. Let us first show that $\omega(x)$ contains a dense orbit. To prove this we use a slight adaptation of a well-known argument showing that if a map is transitive on a compact set $X$, then there is a dense subset of $X$ consisting of points with dense orbit in $X$. Since $\omega(x)$ is compact, there exists a collection of open subsets $U_{n}$ of $M$ such that $\bigcap_{n \geq 1} \bigcup_{k \geq n} U_{n}=\omega(x)$ and such that $\operatorname{diam}\left(U_{n}\right) \rightarrow 0$. By the definition of $\omega(x)$, for each non-empty open subset $U, V$ intersecting $\omega(x)$ there exists $m \geq 0$ such that $f^{-m}(U) \cap V \neq \emptyset$. So $\bigcup_{m<0} f^{m}\left(U_{n}\right) \cap \omega(x)$ is dense in $\omega(x)$ (and of course open). So by Baire, $X=\bigcap_{n \geq 0} \bigcup_{m<0} f^{m}\left(U_{n}\right) \cap \omega(x)$ is also dense, and in particular non-empty. Points in $X$ have dense orbits in $\omega(x)$. It follows that either $x$ is eventually mapped to a periodic point $y$, or that $\omega(x)$ contains a non-periodic recurrent point $y$.

Let us distinguish three cases.

(I) The point $y$ is a non-periodic recurrent point, and there exist arbitrarily small periodic intervals $J$ around $y$. If $x$ is not contained in the orbit of the periodic intervals around $y$, then the domain of the first return map to a small neighbourhood of $x$ is empty, and the assertion is trivial. So we may as well assume that $y=x$. Let $\phi$ be the first return map to $J$. Because of Lemma 2$] \quad J \rightarrow J$ has good distortion properties (because the pullback of a definite neighborhood of $J$ has bounded intersection multiplicity). In particular, the derivative of $\phi$ is bounded by some universal number. Let $P$ be the set of fixed points of $\phi: J \rightarrow J$, and let $\hat{I}_{0}$ be the component of $J \backslash \phi^{-1}(P)$ containing $x$. It is not hard to see that if $\phi^{2}(x) \notin \hat{I}_{0}$, then the domain $\hat{I}_{1}$ of the first return map to $\hat{I}_{0}$ containing $x$ is well-inside $\hat{I}_{0}$. If $\phi^{2}(x) \in \hat{I}_{0}$, then define $\hat{I}_{i} \ni x$ inductively as before. Either

(Ia) for each $i, \phi^{2 i}(x) \in \hat{I}_{0}$; in this case $x$ is contained in a periodic interval $J_{1} \subset \hat{I}_{0} \subset J$ of period two, i.e., with $\phi^{2}\left(J_{1}\right) \subset J_{1}$ and $\phi^{2}\left(\partial J_{1}\right) \subset \partial J_{1}$, or

(Ib) there exists (a minimal) $k \geq 0$ such that $\phi^{2}(x) \notin \hat{I}_{k}$. As before, it is not hard to see that this implies that $\hat{I}_{k+1}$ is $\xi_{0}$-well-inside $\hat{I}_{k}$, with $\xi_{0}$ universal.

In case (Ia), $I:=K_{0}:=I_{0}$ and $J:=K_{1}:=J_{1}$ are both periodic nice intervals of $\phi$-period 1 , respectively 2 , while in case (Ib) $I:=I_{0}$ and $J:=J_{1}$ are nice intervals. The required properties hold for these intervals. To show that either (1) or (2) from the statement of Theorem $\mathrm{A}^{\prime}$ holds, replace $\phi: J \rightarrow J$ by $\phi^{2}: J_{1} \rightarrow J_{1}$ in the above if case (Ia) holds, and repeat the same argument. It follows that either $f$ is Feigenbaum-like at $x$ (from some moment only case (Ia) occurs), or we can take infinitely small nice intervals $J \subset I$ with $J=\mathcal{L}_{x}(I)$.

(II) The point $y$ is a non-periodic recurrent point, and the period of periodic intervals $J_{i} \ni y$ is bounded (so $f$ is finitely often renormalizable at $y$ ). Then let $J$ be the smallest periodic interval containing $y$ (possibly $J=M$ ), let $\phi$ be the first return map to $J$ and $P$ be the set of fixed points of $\phi: J \rightarrow J$. Let $I_{0}$ be the component of $J \backslash \phi^{-1}(P)$ containing $y$. Then the sequence of return maps $\phi_{i+1}: I_{i+1} \rightarrow I_{i}$ around $y$ (defined above) has infinitely many non-central returns (because $y$ is non-periodic), and so by Theorem A there exists $\xi_{0}>0$ and arbitrarily small nice intervals $I^{\prime}$ containing $y$ such that all its first return domains are $\xi_{0}$-wellinside $I^{\prime}$. Notice that $I=\mathcal{L}_{x}\left(I^{\prime}\right)$ is nice, and that $I$ is small if $I^{\prime}$ is small. Let $z \in I$, and let $t(z)>0$ be minimal so that $z^{\prime}=f^{t(z)}(z) \in I^{\prime}$. Then $\mathcal{L}_{z}(I)$ is contained in the pullback by $f^{t(z)}$ of $\mathcal{L}_{z^{\prime}}\left(I^{\prime}\right)$. It follows that for each $z$ its first return domain $\mathcal{L}_{z}(I)$ to $I$ is $\xi_{0}^{\prime}$-well-inside $I$. (Of course it is conceivable that the domain of the first return map to $I$ is empty, in which case the assertion of the Theorem is trivial.) 
(III) $y$ is a (repelling) periodic point with period $p$. Let $\lambda=D f^{p}(y)$ be its multiplier.

Claim 1: There exists $\xi_{0}>0$ and arbitrarily small nice intervals $I^{\prime}$ around $y$ such that each fundamental domain of $R_{I^{\prime}}$ restricted to $\mathcal{L}_{y}\left(I^{\prime}\right)$ is $\xi_{0}$-well-inside $I^{\prime}$. To see this, we first remark that there exist sequences $z_{i} \uparrow y, z_{i}^{\prime} \downarrow y$ of points that are eventually mapped into periodic orbits $O \not \supset y$, respectively $O^{\prime} \not \ngtr y$. Here we take $z_{i}, z_{i}^{\prime}$ so that $f^{p}\left(z_{i+1}\right)=z_{i}$ and $f^{p}\left(z_{i+1}^{\prime}\right)=z_{i}^{\prime}$. If $|\lambda|$ is not large, then neighbouring fundamental domains are comparable in length, so the claim holds for $I^{\prime}=\left(z_{i}, z_{j}^{\prime}\right)$ regardless of the choice for $i$ and $j$ (provided they are large). If $\lambda<0$, then $I^{\prime}:=\left(z_{i}, f^{p}\left(z_{i}\right)\right)$ is the required nice interval: each fundamental domain of $R_{I^{\prime}}$ is well-inside $I^{\prime}$ (because we assumed that $|\lambda|$ is large, this is trivial). If $\lambda>0$ is large, then choose $j$ minimal so that $\left|z_{j}^{\prime}-y\right|<\left|z_{i}-y\right|$. If $\left|z_{j}^{\prime}-y\right| \geq 10\left|z_{i+1}-y\right|$, then take $I^{\prime}=\left(z_{i}, z_{j}^{\prime}\right)$ and otherwise take $I^{\prime}=\left(z_{i+1}, z_{j}^{\prime}\right)$. In both cases $\mathcal{L}_{y}\left(I^{\prime}\right)$ is well-inside $I^{\prime}$, so again the claim holds.

Claim 2: There exists $\xi_{1}$ so that provided we take $I^{\prime}$ sufficiently small, for each $z \in I^{\prime} \backslash \mathcal{L}_{y}\left(I^{\prime}\right), \quad \mathcal{L}_{z}\left(I^{\prime}\right)$ is $\xi_{1}$-well-inside $I^{\prime}$. To see this, take two intervals $I^{\prime} \subset I^{\prime \prime}$ as above with $I^{\prime}$ well-inside $I^{\prime \prime}$ and so that for some $t \geq 1, f^{t}: I^{\prime} \rightarrow I^{\prime \prime}$ is a diffeomorphism. Some iterate $z^{\prime}$ of $z$ will enter $I^{\prime \prime} \backslash \mathcal{L}_{y}\left(I^{\prime \prime}\right)$ before returning to $I^{\prime}$. Since $I^{\prime}$ is well-inside $I^{\prime \prime}$, by Proposition 1, $\mathcal{L}_{z^{\prime}}\left(I^{\prime}\right)$ is well-inside $I^{\prime \prime}$ (and contained in the fundamental domain $\left.I^{\prime \prime} \backslash \mathcal{L}_{y}\left(I^{\prime \prime}\right)\right)$. If $|\lambda|$ is not large, then $\mathcal{L}_{z^{\prime}}\left(I^{\prime}\right)$ is well-inside two neighbouring fundamental domains, and so it follows that $\mathcal{L}_{z}\left(I^{\prime}\right)$ is well-inside $I^{\prime}$. If $|\lambda|$ is large, then $f^{t} \mid I^{\prime}$ has bounded distortion, and therefore $\mathcal{L}_{z}\left(I^{\prime}\right)=\left(f^{t} \mid I^{\prime}\right)^{-1}\left(\mathcal{L}_{z^{\prime}}\left(I^{\prime}\right)\right.$ is well-inside $I^{\prime}$.

Now taking $I=\mathcal{L}_{x}\left(I^{\prime}\right)$ the required statements hold (here we use that $x$ is preperiodic, but not periodic, which implies that the return domains of $I$ are pullbacks of return domains to $I^{\prime}$ that are contained in $\left.I^{\prime} \backslash \mathcal{L}_{y}\left(I^{\prime}\right)\right)$. So this completes the case that $y$ is periodic.

The final part of the assertion of Theorem $\mathrm{A}^{\prime}$ holds because both $I$ and $J$ are nice.

\section{Theorem C: Improved Koebe Principles AND NEGATIVE SChWARZIAN DERIVATIVE}

Before proving Theorem B, we shall prove Theorem C. First we state and prove an improved Koebe Principle. The usual Koebe Lemma applies to a situation when one has an interval $T_{0} \supset J$ such that $f_{\mid T_{0}}^{n}$ is a diffeomorphism, and such that either, in addition, $T_{0}, \ldots, f^{n}\left(T_{0}\right)$ are pairwise disjoint or that the Schwarzian derivative of $f$ is negative. In the following version we do not require that $T_{0}, \ldots, f^{n}\left(T_{0}\right)$ are pairwise disjoint, have finite total length or even that $f_{\mid T_{0}}^{n}$ is a diffeomorphism, but only an upper bound for $\sum_{i=0}^{n-1}\left|f^{i}(J)\right|$.

Proposition 2 (Koebe Principle requiring less disjointness). Assume that $f$ is $C^{2}$. Then there exist constants $K>0$ and a function $O(\epsilon)$ with $O(\epsilon) \rightarrow 0$ as $\epsilon \downarrow 0$ with the following properties. Let $n$ be an integer and $J$ an interval such that $f_{\mid J}^{n}$ is a diffeomorphism. Let $T$ be a $\delta$-scaled neighbourhood of $f^{n}(J)$ for some $\delta>0$ and $T_{0}, \ldots, T_{n}:=T$ be the pullback of $T$ along $J, \ldots, f^{n}(J)$. 
- Let $\mathcal{N} \subset\{0, \ldots, n-1\}$ be the set of integers $i$ for which $T_{i}$ contains a critical point and let $\epsilon=\max \left|T_{i}\right|$. Then for each $x, y \in J$,

$$
\frac{\left|D f^{n}(x)\right|}{\left|D f^{n}(y)\right|} \leq \exp \left(O(\epsilon) \sum_{i=0}^{n-1}\left|f^{i}(J)\right|\right) \cdot\left[\frac{1+\delta}{\delta}\right]^{2} \cdot \exp \left(K \cdot \sum_{m \in \mathcal{N}} \frac{\left|f^{m}(J)\right|}{\operatorname{dist}\left(f^{m}(J), \mathbf{C r}\right)}\right)
$$

(If there are no such integers $i$, then we take the sum $\sum_{m \in \mathcal{N}}$ above to be equal to zero.) If $f$ is $C^{3}$, then there exists $\hat{K}>0$ such that $O(t) \leq \hat{K} t$ for each $t>0$.

- If $f_{\mid T}^{n}$ is a diffeomorphism, then $T$ is a $\delta^{\prime}\left(\epsilon, \sum_{i=0}^{n-1}\left|f^{i}(J)\right|\right)$-scaled neighbourhood of $J$. Here $\delta^{\prime}$ is a function which is specified in the proof of the proposition.

In the proof of this proposition we need to use the following cross-ratios; see [17. Let $j \subset t$ be intervals, and let $l, r$ be the components of $t \backslash j$. Define

$$
A(t, j)=\frac{|t| \cdot|j|}{|l \cup j| \cdot|j \cup r|} \text { and } B(t, j)=\frac{|t| \cdot|j|}{|l| \cdot|r|}
$$

and if $f_{\mid T}$ is monotone, define

$$
A(f, t, j)=\frac{A(f(t), f(j))}{A(t, j)} \text { and } B(f, t, j)=\frac{B(f(t), f(j))}{B(t, j)} .
$$

It is well known that if $f$ is $C^{2}$, under the assumption stated above, see [17] and also 22], there exists a function $O(\epsilon)$ with $O(\epsilon) \rightarrow 0$ as $\epsilon \downarrow 0$, so that for any intervals $j \subset$ $t$ as above with $f_{\mid t}$ a diffeomorphism, $A(f, t, j) \geq \exp (-|r| \cdot O(|l|))$ and $B(f, t, j) \geq$ $\exp (-|t| \cdot O(|t|))$. If $f$ is $C^{3}$, there is even a universal constant $C>0$ so that $A(f, t, j) \geq \exp (-C \cdot|l| \cdot|r|))$ and $B(f, t, j) \geq \exp \left(-C|t|^{2}\right)$.

Proof. The main idea of this lemma appeared before in 22 (for example, equation (2.5) in the proof of Theorem 2.3 in that paper); see also [5]. Let $\epsilon>0$ be such that each interval $T_{0}, \ldots, T_{n}$ has length at most $\epsilon$. Take any point $x$ in the interior of $J$. The point $x$ divides the intervals $J$ into two intervals $J^{-}, J^{+}$. Similarly split $J_{i}:=f^{i}(J)$ into $J_{i}^{+}=f^{i}\left(J^{+}\right)$and $J_{i}^{-}=f^{i}\left(J^{-}\right)$and also split $T_{i}$ into $T_{i}^{-} \supset$ $J_{i}^{-}, T_{i}^{+} \supset J_{i}^{+}$. We either have

$$
\frac{\left|f^{n}\left(J^{-}\right)\right|}{\left|J^{-}\right|} \geq \frac{\left|f^{n}(J)\right|}{|J|} \text { or } \frac{\left|f^{n}\left(J^{+}\right)\right|}{\left|J^{+}\right|} \geq \frac{\left|f^{n}(J)\right|}{|J|} .
$$

Without loss of generality, suppose the latter holds. Let $n_{s}<\cdots<n_{1}<n_{0}:=n$ be the integers $i$ such that $T_{i}^{-}$contains a critical point. Fix $i \in\{1, \ldots, s\}$ and take $r=J_{n_{i}+1}^{+}, l=T_{n_{i}+1}^{-} \cup J_{n_{i}+1}^{-}, j=\left\{f^{n_{i}+1}(x)\right\}$ and $t=l \cup j \cup r$. Applying $A$ to $t=l \cup j \cup r$ and $j$ gives

$$
\begin{aligned}
A\left(f^{n_{i-1}-n_{i}-1}, l \cup j \cup r, j\right) & \geq \exp \left(-\sum_{k=0}^{n_{i-1}-n_{i}-1}\left|f^{k}(r)\right| \cdot O\left(\left|f^{k}(t)\right|\right)\right) \\
& \geq C_{0}\left(n_{i}, n_{i-1}\right),
\end{aligned}
$$


where $C_{0}(a, b):=\exp \left(-O(\epsilon) \cdot \sum_{k=a}^{b-1}\left|f^{k}(J)\right|\right)$. Using the definition of the crossratio $A$, and that $|t| \geq|l|$,

$$
\left|D f^{n_{i-1}-n_{i}-1}\left(f^{n_{i}+1}(x)\right)\right| \geq C_{0}\left(n_{i-1}, n_{i}\right) \cdot \frac{\left|f^{n_{i-1}}\left(J^{+}\right)\right|}{\left|f^{n_{i}+1}\left(J^{+}\right)\right|} \cdot \frac{\left|T_{n_{i-1}}^{-} \cup J_{n_{i-1}}^{-}\right|}{\left|T_{n_{i-1}}^{-} \cup J_{n_{i-1}}^{-} \cup J_{n_{i-1}}^{+}\right|} .
$$

By the non-flatness of the critical points, there exists a universal constant $K_{2}>0$ such that

$$
\left|D f\left(f^{n_{i}}(x)\right)\right| \geq \frac{\left|f^{n_{i}+1}\left(J^{+}\right)\right|}{\left|f^{n_{i}}\left(J^{+}\right)\right|} \cdot \exp \left(-K_{2} \cdot \frac{\left|J_{n_{i}}\right|}{\operatorname{dist}\left(J_{n_{i}}, \mathbf{C r}\right)}\right),
$$

where $\mathbf{C r}$ is the set of critical points. Also,

$$
\frac{\left|T_{n_{i-1}}^{-} \cup J_{n_{i-1}}^{-}\right|}{\left|T_{n_{i-1}}^{-} \cup J_{n_{i-1}}^{-} \cup J_{n_{i-1}}^{+}\right|} \geq \frac{\operatorname{dist}\left(J_{n_{i-1}}, \mathbf{C r}\right)}{\operatorname{dist}\left(J_{n_{i-1}}, \mathbf{C r}\right)+\left|J_{n_{i-1}}\right|} \geq \exp \left(-K_{3} \frac{\left|J_{n_{i-1}}\right|}{\operatorname{dist}\left(J_{n_{i-1}}, \mathbf{C r}\right)}\right) \text {. }
$$

Combining all these inequalities,

$$
\begin{aligned}
\left|D f^{n}(x)\right| \geq C_{0}(0, n) \cdot \frac{\left|f^{n}\left(J^{+}\right)\right|}{\left|J^{+}\right|} \cdot \frac{\left|T_{n_{0}}^{-} \cup J_{n_{0}}^{-}\right|}{\left|T_{n_{0}}^{-} \cup J_{n_{0}}^{-} \cup J_{n_{0}}^{+}\right|} & \\
& \cdot \prod_{i=1}^{s} \exp \left(-\left(K_{2}+K_{3}\right) \cdot \frac{\left|J_{n_{i}}\right|}{\operatorname{dist}\left(J_{n_{i}}, \mathbf{C r}\right)}\right) .
\end{aligned}
$$

By assumption $\frac{\left|f^{n}\left(J^{+}\right)\right|}{\left|J^{+}\right|} \geq \frac{\left|f^{n}(J)\right|}{|J|}$. Hence, by the definition of $\delta$ in the statement of the theorem, and by the disjointness of the orbit of $J$, we obtain

$$
\left|D f^{n}(x)\right| \geq C_{0}(0, n) \cdot \frac{\delta}{1+\delta} \cdot \frac{\left|f^{n}(J)\right|}{|J|} \cdot \exp \left(-K \cdot \sum_{m \in \mathcal{N}} \frac{\left|f^{m}(J)\right|}{\operatorname{dist}\left(f^{m}(J), \mathbf{C r}\right)}\right) .
$$

Let us now prove the reverse inequality. To do this, we consider the cross-ratio $B$. Again, either

$$
\frac{\left|f^{n}\left(J^{-}\right)\right|}{\left|J^{-}\right|} \leq \frac{\left|f^{n}(J)\right|}{|J|} \text { or } \frac{\left|f^{n}\left(J^{+}\right)\right|}{\left|J^{+}\right|} \leq \frac{\left|f^{n}(J)\right|}{|J|} .
$$

Suppose the first holds and take an interval $V \subset J^{-}$. Since

$$
B\left(f^{n}, J^{-}, V\right) \geq \exp \left(-O\left(\max _{i \leq n}\left|f^{i}(J)\right|\right) \cdot \sum\left|f^{i}(J)\right|\right) \geq C_{0}(0, n),
$$

by taking $V$ converging to $J^{-}$we get that

$$
\left|D f^{n}(x)\right| \leq C_{0}(0, n) \frac{1}{\left|D f^{n}(a)\right|} \cdot\left(\frac{\left|f^{n}\left(J^{-}\right)\right|}{\left|J^{-}\right|}\right)^{2},
$$

where $a$ is the boundary point of $J^{-}$that is not inside $J$. Using (4) (with $x=a$ ) to bound $\left|D f^{n}(a)\right|$ and using $\frac{\left|f^{n}\left(J^{-}\right)\right|}{\left|J^{-}\right|} \leq \frac{\left|f^{n}(J)\right|}{|J|}$, we get (3)).

To prove the second part of the proposition, choose $j, l, r \subset T$ such that $l \cup j=J$, $\left|f^{n}(l)\right|=\left|f^{n}(j)\right|$ and such that $f^{n}(r)$ is one of the components of $f^{n}(T \backslash J)$. As before, $A\left(f^{n}, t, j\right) \geq C_{0}(0, n)$. Using the definition of $A$ and $\delta$ then gives

$$
\frac{|r \cup j|}{|j|} \geq \frac{|l \cup j|}{|j|} \frac{|r \cup j|}{|t|} \geq C_{0} \frac{2(1+\delta)}{1(2+\delta)} .
$$


Since $C_{0}=\exp (-x)$ with $x=O(\epsilon) \sum_{i=0}^{n-1}\left|f^{i}(J)\right|$ and $\exp (-x) \geq 1-x$ for $x \geq 0$, this gives $\frac{|r|}{|j|} \geq \frac{-2 x+\delta(1-2 x)}{2+\delta}$. Since by the first part of this proposition, the length of $l \cup j$ is at most $2 \frac{1}{C_{0}}[(1+\delta) / \delta]^{2}$ times the length of $j$, the result follows.

Let us now prove that the Schwarzian derivative of high iterates of $f$ is negative. In order to do this, we assume that $f$ is $C^{3}$ and prove the following proposition.

Proposition 3. Assume that $f$ is $C^{3}$. For each integer $N$, and each $\xi>0, \delta>0$, $S>0$, there exists $\tau>0$ with the following property. Let $n$ be an integer and $J$ an interval such that $\sum_{0 \leq i<n}\left|f^{i}(J)\right| \leq S$. Let $T_{n}$ be a $\delta$-scaled neighbourhood of $f^{n}(J)$, and let $T_{0}, \ldots, T_{n}$ be the pullback with $T_{i} \supset f^{i}(J)$. Let $\mathcal{N} \subset\{0, \ldots, n-1\}$ be the set of integers for which $T_{i}$ contains a critical point. Assume that

- $\# \mathcal{N} \leq N$

- $\left|f^{n}(J)\right| \geq \xi \cdot \operatorname{dist}\left(f^{n}(J), \mathbf{C r}\right)$, where $\mathbf{C r}$ is the set of critical points;

- $\left|f^{n}(J)\right| \leq \tau$.

Then

$$
S f^{n+1}(x)<0 \text { for all } x \in J .
$$

Proof. Since $f$ is $C^{3}$ and all its critical points are non-flat, there exists $C>0$ such that $S f(x)<C$ for all $x$, and there exists a neighbourhood $U$ of $\mathbf{C r}$ and a constant $C^{\prime}>0$ with

$$
S f(y)<-\frac{C^{\prime}}{[\operatorname{dist}(y, \mathbf{C r})]^{2}} \text { for all } y \in U .
$$

Let $n_{s}<\cdots<n_{0}=n$ be the integers $m$ so that $\left|f^{m}(J)\right| \geq \xi \cdot \operatorname{dist}\left(f^{m}(J), \mathbf{C r}\right)$ (for example, when $f^{m}(J)$ contains a critical point). Because of the composition formula for the Schwarzian derivative, it suffices to show the following claim for each $i \leq s$.

Claim: $S f^{k_{i}}(x)<0$ for any $x \in f^{n_{i+1}+1}(J)$ where $k_{i}=n_{i}-n_{i+1}$, where $k_{0}=n_{s}+1$ and $n_{s+1}=-1$.

Proof of Claim. Fix $i$, write $n^{\prime}=k_{i}$ and let us show that $S f^{n^{\prime}}(y)<0$ for any $y \in f^{n_{i+1}+1}(J)$. By the composition formula for the Schwarzian derivative,

$S f^{n^{\prime}}(y)=\left|D f^{n^{\prime}-1}(y)\right|^{2} \cdot\left(S f\left(f^{n^{\prime}-1}(y)\right)+\sum_{i=0}^{n^{\prime}-2} S f\left(f^{i}(y)\right) \cdot\left|D f^{n^{\prime}-1-i}\left(f^{i}(y)\right)\right|^{-2}\right)$.

From (5) this is at most

$$
\left|D f^{n^{\prime}-1}(y)\right|^{2} \cdot\left(\frac{-C^{\prime}}{\left[\operatorname{dist}\left(f^{n^{\prime}-1}(y), \mathbf{C r}\right)\right]^{2}}+\sum_{i=0}^{n^{\prime}-2}\left(C \cdot\left|D f^{n^{\prime}-1-i}\left(f^{i}(y)\right)\right|^{-2}\right)\right) .
$$

Because of the assumptions and from the previous proposition, this is at most

$$
\begin{gathered}
\leq\left|D f^{n^{\prime}-1}(y)\right|^{2} \cdot\left(\frac{-C^{\prime} \xi^{2}}{\left|f^{n_{i-1}}(J)\right|^{2}}+C^{\prime \prime} \sum_{j=n_{i}}^{n_{i-1}-1} \frac{\left|f^{j}(J)\right|^{2}}{\left|f^{n_{i-1}}(J)\right|^{2}}\right) \\
=\frac{\left|D f^{n^{\prime}-1}(y)\right|^{2}}{\left|f^{n_{i-1}}(J)\right|^{2}}\left(-C^{\prime} \xi^{2}+C^{\prime \prime} \sum_{j=n_{i}}^{n_{i-1}-1}\left|f^{j}(J)\right|^{2}\right) .
\end{gathered}
$$


Here $C^{\prime \prime}$ depends on the cardinality of $\mathcal{N}, C$ and on $\xi$ and $S$ (through the exponential term in (3)). Provided $\left|f^{n}(J)\right|$ is sufficiently small, all $\left|f^{i}(J)\right|, 0 \leq i<n$, will be small (because there are no wandering intervals). Because of this, and by the bound for $\sum_{0<i<n}\left|f^{i}(J)\right|$, we get $S f^{n^{\prime}}(y)<0$ for $y \in f^{n_{i+1}+1}(J)$. Combining this, and using that compositions of maps with negative Schwarzian again have negative Schwarzian, completes the proof.

6.1. Proof of Theorem C. Part (1) of Theorem C is already proved in Proposition 1. Before proving part (2), let us prove part (3). According to Theorem A' there are two cases:

Case 1. The critical point $c$ is not in the basin of a periodic attractor (so in particular non-periodic) and $f$ is not of Feigenbaum type at $c$. According to Theorem $\mathrm{A}^{\prime}$ there exists (an arbitrarily small) nice interval $T$ containing $c$ with the property that each first return to $T$ is well-inside $T$. (Here we use that $c$ is not in the basin of a periodic attractor.) Let $\mathcal{L}_{y}(T)$ be the component of the domain of this first entry map to $T$ containing $y$ (this set can be empty). Let $V \subset T$ be $\mathcal{L}_{c}(T)$ or if that is empty, let $V$ be any neighbourhood of $c$ that is well-inside $T$. Now take any $x$ so that, for some $n, f^{n}(x) \in V$. We want to show that $S f^{n+1}(x)<0$. Let $\tilde{n} \leq n$ be minimal such that $f^{\tilde{n}}(x) \in T$ and either (i) one of the intervals in the pullback of $\mathcal{L}_{f^{\tilde{n}}(x)}(T)$ along $\left\{x, \ldots, f^{\tilde{n}}(x)\right\}$ contains a critical point or (ii) so that $f^{\tilde{n}}(x) \in V$. Since $f^{n}(x) \in V$, such an integer exists. If (i) holds, then define $J=\mathcal{L}_{f \tilde{n}(x)}(T)$ and if (ii) holds, then define $J=V$. In both cases, $J$ is well-inside $T$, and one of the intervals in its pullback along $\left\{x, \ldots, f^{\tilde{n}}(x)\right\}$ contains a critical point. Take $\hat{n}$ maximal with $0 \leq \hat{n}<\tilde{n}$ such that $f^{\hat{n}}(x) \in T$ (if $\hat{n}$ does not exist, let $\hat{n}=0)$. Let $J_{0}, \ldots, J_{\tilde{n}}$ and $T_{0}, \ldots, T_{\tilde{n}}$ be the pullbacks of, respectively, $J$ and $T$ along $\left\{x, \ldots, f^{\tilde{n}}(x)\right\}$, and let $n^{\prime}$ be minimal such that $J_{n^{\prime}}$ contains a critical point. Note that $\hat{n}<n^{\prime} \leq \tilde{n} \leq n$ and that $T_{\hat{n}}=\mathcal{L}_{f^{\hat{n}}(x)}(T)$. By the minimality of $\tilde{n}$, none of the intervals $T_{0}, \ldots, T_{\hat{n}-1}$ contains a critical point; moreover, $T_{\hat{n}}, \ldots, T_{n^{\prime}-1}$ is pairwise disjoint and the same holds for $J_{\hat{n}}, \ldots, J_{n^{\prime}-1}$. In particular, since $J$ is well-inside $T$, also $J_{n^{\prime}}$ is well-inside $T_{n^{\prime}}$. Finally, by definition, $J_{n^{\prime}}$ contains a critical point. From all this and Proposition 3, it follows that $S f^{n^{\prime}+1}(x)<0$. If $n^{\prime}=n$, then we are done. If $n^{\prime}<n$, then define $x^{\prime}=f^{n^{\prime}+1}(x)$ and repeat the argument. Since the composition of maps with negative Schwarzian derivative again has negative Schwarzian derivative, we are done. This completes the proof of part (3) of Theorem $\mathrm{C}$ in this case.

Case 2. The critical point $c$ is of Feigenbaum type (as defined in Theorem $\mathrm{A}^{\prime}$ ). Let $\left\{K_{i}\right\}_{i=0}^{\infty}$ be a nested sequence of nice $p 2^{i}$-periodic intervals containing $c$. We want to show that if $f^{n}(x) \in K_{i+1}$ where $i$ is large, then $S f^{n+1}(x)<0$. For the moment fix $i$ and let $n^{\prime} \leq n$ be minimal such that $f^{n^{\prime}}(x) \in K_{i+1}$, and let $\hat{n} \leq n^{\prime}$ be minimal such that $f^{\hat{n}} \in K_{i}$. Then $n^{\prime}=\hat{n}+k p 2^{i}$ for some integer $k$. If $k \leq 2$, then the intersection multiplicity of the pullback of $K_{i}$ along $\left\{x, \ldots, f^{\hat{n}^{\prime}}(x)\right\}$ is at most 3 , and so we can apply the previous proposition, replacing $n$ by $n^{\prime}$ and taking $f^{n^{\prime}}(J)=K_{i+1}$ and $T_{n^{\prime}}=K_{i}$ (provided we take $i$ sufficiently large). If $k>2$, then $f^{\hat{n}+(k-2) p 2^{i}}(x)$ is contained in a fundamental domain $F_{i}$ associated to the repelling fixed point of $f^{p 2^{i}}: K_{i} \rightarrow K_{i}$ in $\partial K_{i}$. Because of the corollary at the end of Section 2 this fundamental domain is comparable in size to $K_{i}$ (here we use that $f^{\hat{n}+k p 2^{i}}(x) \in K_{i+1}$ and that $K_{i+1}$ is not small compared to $K_{i}$ in this Feigenbaum situation). Moreover, it is comparable in size to its two neighbouring fundamental 
domains $F_{i, l}, F_{i, r}$. So replacing in the proposition $n$ by $\tilde{n}:=\hat{n}+(k-2) p 2^{i}$ and taking $f^{\tilde{n}}(J)=F_{i}$ and $T_{\tilde{n}}=F_{i, l} \cup F_{i} \cup F_{i, r}$, we again obtain that $S f^{\tilde{n}+1}(x)<0$ provided $i$ is sufficiently large.

Part (2) of Theorem $\mathrm{C}$ now follows easily: let $U$ be a nice neighbourhood of a critical point as in part (3) such that $f^{n}(T) \subset U$. Since $U$ is nice and $f^{n} \mid T$ is a diffeomorphism, $T, \ldots, f^{n}(T)$ are either contained in $U$ or outside $U$. Let $s$ be maximal with $0 \leq s<n$ such that $f^{s}(T) \subset U$. If there exists no such $s$, then $T, \ldots, f^{n}(T)$ are disjoint and the result follows from the previous proposition. If there exists such an integer $s$, then $f^{s+1}: T \rightarrow f^{s+1}(T)$ has negative Schwarzian. By a theorem of Mañé [13] any compact forward-invariant set not containing parabolic or critical points, is hyperbolic. Combined, this implies that $f^{s+1}: T \rightarrow f^{s+1}(T)$ and $f^{n-s-1}: f^{s+1}(T) \rightarrow f^{n}(T)$ have bounded distortion, and we obtain part (2). (In fact, one can derive this result of Mañé also from our real bounds.)

\section{Theorem B: LARGe Bounds}

Let us now prove large bounds. This will be done in the two propositions in this section. If $I$ is a nice interval containing a non-periodic recurrent critical point $c$, then we say that $J$ is a child of $I$ if $J$ is a component of $f^{-1}(J)$ where $J^{\prime}$ is a diffeomorphic pullback of $I$ and $J$ contains a critical point $c^{\prime}$ with $\omega(c)=\omega\left(c^{\prime}\right) \ni$ $c, c^{\prime}$.

Let us first prove part (1) of Theorem B. It will be convenient to define $\mathcal{L}_{x}(J)$ to be the domain of the first entry map to $J$ containing $x$, and to define inductively $\mathcal{L}_{x}^{i+1}(J)=\mathcal{L}_{x}\left(\mathcal{L}_{x}^{i}(J)\right)$.

Proposition 4. For each $C>0, \delta>0$ there exists $N^{\prime}$ such that if a nice interval $I$ has at least $N^{\prime}$ children $K_{1} \supset K_{2} \supset \cdots$ around some critical point, and has each of its domains in $I \delta$-well-inside $I$, then for $I^{\prime}=K_{N}$ with $N \geq N^{\prime}$ each of the first return domains to $I^{\prime}$ is $C$-well inside $I^{\prime}$.

Note that because of Theorem A(1) there exist many nice intervals $I$ such that each of the domains of the first return map to $I$ is $\delta$-well-inside $I$ for some $\delta>0$.

Proof. Assume that $I$ is a nice interval as above, and let $R_{I}$ be the first entry map to $I$. Let $s(i)$ be such that $K_{i}$ is a unimodal (or homeomorphic if $d$ is an inflection point) pullback of $I$ under $f^{s(i)}$. Then $f^{s(i)}\left(K_{i+1}\right)$ is contained in a first return domain to $I$. It follows that $K_{i+1}$ is $\delta^{\prime \prime}$-well-inside $K_{i}$ for each $i=1, \ldots, N$.

Next consider a point $u \in I^{\prime}=K_{N}$ in a return domain to $I^{\prime}$, and let $f^{s}$ be the unimodal (or homeomorphic) map such that $K_{N}$ is a component of $f^{-s}(I)$ (this is possible because $K_{N}$ is a child of $\left.I\right)$. Note that $f\left(K_{N}\right), \ldots, f^{s-1}\left(K_{N}\right)$ do not intersect $I^{\prime}=K_{N}$ (otherwise, $K_{N}$ could not be a unimodal pullback of $I$ ). Let $v=f^{s}(u) \in I$. If $v \in I^{\prime}$, then $I \supset K_{1} \supset K_{2} \supset \cdots \supset K_{N}:=I^{\prime}$ and since $I^{\prime}$ is very deep inside $I$ we can pull back this space through the unimodal map $f^{s}: I^{\prime} \rightarrow I$. If $v \notin I^{\prime}$, then pick an integer $i \in\{1, \ldots, N\}$, let $R_{K_{i}}$ be the first entry map to $K_{i}$ and let $x_{i}=R_{K_{i}}(v)$. Then applying Theorem B(1) (taking $I$ to be $K_{i}$ and $V$ to be $K_{i+1}$ ) shows that $\mathcal{L}_{x_{i}}\left(K_{i+1}\right)$ is well-inside $K_{i}$, and so by Koebe we get that $\mathcal{L}_{v}\left(K_{i+1}\right)$ is $\tilde{\delta}$ well-inside $\mathcal{L}_{v}\left(K_{i}\right)$. Hence $\mathcal{L}_{u} \mathcal{L}_{v}\left(K_{i+1}\right)$ is $\tilde{\delta}^{\prime}$-well-inside $\mathcal{L}_{u} \mathcal{L}_{v}\left(K_{i}\right)$. Since this holds for $i=1, \ldots, N$, and since $\mathcal{L}_{u} \mathcal{L}_{v}\left(K_{1}\right) \subset I^{\prime}$ and $\mathcal{L}_{u} \mathcal{L}_{v}\left(K_{N}\right)=\mathcal{L}_{u} \mathcal{L}_{v}\left(I^{\prime}\right)=\mathcal{L}_{u}\left(I^{\prime}\right)$ (because $v \notin I^{\prime}$ ), it follows that $\mathcal{L}_{u}\left(I^{\prime}\right)$ is very deep inside $I^{\prime}$ when $N$ is large. 
Let $J$ be a nice interval, and let $J_{1}$ be its first return domain containing a critical point $c$. Define $J_{i}=\mathcal{L}_{c}^{i}(J)$. Assume that $k$ is maximal such that the first return $\operatorname{map} \phi_{J_{i-1}}(c) \in J_{i}$ for $1 \leq i<k$ and $\phi_{J_{k-1}}(c) \notin J_{k}$, and define $\mathcal{C}_{c}(J)=J_{k}$ (so that $\mathcal{C}_{c}(J)$ is the next non-central return in the cascade). In addition, we say that a pair of nice intervals $W \supset V$ is nice if no forward iterate of $\partial V$ enters the interior of $W$. Note that any pullback $W^{\prime} \supset V^{\prime}$ of a nice pair $W \supset V$ is again a nice pair.

Lemma 15. Let $J \subset W$ be a return domain to $W$ that contains a point $c$ and $J \cap U=\emptyset$. Then any pullback of $U$ that contains $c$ is contained in $\mathcal{C}_{c}(W)$.

Proof. No pullback of $U$ can contain a boundary point of $\mathcal{C}_{c}(W)$, because otherwise $U$ would contain a boundary point of $W$, a contradiction.

Lemma 16. There exists a function $\rho_{1}: \mathbb{R}^{+} \rightarrow \mathbb{R}^{+}$such that $\rho_{1}(\xi) \rightarrow \infty$ as $\xi \rightarrow \infty$ with the following properties. Let $W$ be a nice interval containing a critical point $c$ and let $J=\mathcal{L}_{c}(W)$. Assume that either $c$ is a turning point or that the first return map $\phi: J \rightarrow W$ is monotone. Let $V$ be an interval that is $\xi$-well-inside $W$ and such that $W \supset V$ is a nice pair. Let $s>0$ and assume that $f^{s}(z) \in V$ (not necessarily for the first time). Denote by $V_{i}, W_{i}$ the pullback of $V, W$ along $f^{i}(z), \ldots, f^{s}(z)$. Then there exists $0 \leq t<s$ and $W_{t}^{1}$ with $V_{t} \subset W_{t}^{1} \subset \mathcal{L}_{f^{t}(z)} W$ and such that

(1) $V_{t}$ is $\rho_{1}(\xi)$-well-inside $W_{t}^{1}$, and if $t>0, V_{t} \subset W_{t}$ is a nice pair, and

(2) each pullback of $W_{t}^{1}$ intersecting $c$ is contained in $\mathcal{C}_{c}(W)$.

Proof. Observe that by part (2) of Theorem C, there exists a function $\hat{\rho}$ with $\hat{\rho}(\xi) \rightarrow \infty$ as $\xi \rightarrow \infty$ so that the pullback of $V$ by any first entry map to $W$ is $\rho(\xi)$-well-inside its domain. So we can assume that $z \in W$. For simplicity assume $\xi^{\prime}:=\rho(\xi)<\xi$ and that $\xi$ is large.

Let $0:=t_{0}<t_{1}<t_{2}<\cdots<t_{k}=s$ be the integers such that $f^{t_{j}}(z) \in W$. By the above observation $V_{t_{k-1}}$ is $\xi^{\prime}$-well-inside $W_{t_{k-1}}$. Hence, if $f^{t_{k-1}}(z) \notin J$, then by the previous lemma all the required properties hold for $t=t_{k-1}$, taking $W_{t}^{1}=\mathcal{L}_{f^{t}(z)}(W)$ and $\rho_{1}(\xi)=\hat{\rho}(\xi)$. So from now on we assume that $f^{t_{k-1}}(z) \in J$, and let $k^{\prime} \geq 0$ be minimal such that $f^{t_{k^{\prime}}}(z), \ldots, f^{t_{k-1}}(z) \in J$. Let $k^{\prime \prime} \geq k^{\prime}$ be minimal such that $W_{t_{k^{\prime \prime}}} \subset \cdots \subset W_{t_{k-1}}=J \subset W_{t_{k}}=W$. If $W_{t_{k-2}} \not \supset c$, then define $k^{*}=k-2$. If $W_{t_{k-2}} \ni c, k^{\prime \prime}>k^{\prime}$ and if there exists some $i=k^{\prime}, \ldots, k^{\prime \prime}-1$ with $f^{t_{i}}(z) \in W_{t_{k-1}} \backslash W_{t_{k-2}}$, then let $k^{*}$ be the maximal such integer (and otherwise leave $k^{*}$ undefined). We can assume that $J$ is not $\sqrt{\xi}$-well-inside $W$, because otherwise the lemma also holds: if $k^{\prime}>0$, then pull back once more (the required properties hold for $t=t_{k^{\prime}-1}$ setting $W_{t}^{1}=\mathcal{L}_{f^{t}(z)}(W)$ ), whereas if $k^{\prime}=0$, simply set $t=0$ and $W_{t}^{1}=W$. This means that we can also assume that $V \subset W \backslash J$ (since $V \subset W$ is a nice pair; otherwise $V \supset J$, and $J$ would be $\xi$-well-inside $W$ ).

Although we have no control over the distortion of $\phi: J \rightarrow W$, Lemma 2 gives that $\phi: W_{t_{k-2}} \rightarrow J$ is quasi-polynomial: it is the composition of at most \# $\mathbf{C r}$ maps of the type $L \circ f$, where $L$ is a diffeomorphism with universal bounded distortion.

Claim: There exists a function $\rho_{1}$ as above such that $V_{\hat{t}}$ is $\rho_{1}(\xi)$-well-inside $\hat{W}$, where either (i) $\hat{t}=t_{k^{\prime}}$ and $\hat{W}=J$ or (ii) $\hat{t}=t_{k^{\prime \prime}}$ and $\hat{W}=W_{\hat{t}}$, or (iii) $k^{*}$ is defined, $\hat{t}=t_{k^{*}}$ and $\hat{W}=\mathcal{L}_{f^{\hat{t}}(z)}(J)$.

This claim implies the lemma. If (i) holds, take $t=t_{k^{\prime}-1}, W_{t}^{1}=\mathcal{L}_{f^{t}(z)}(W)$ if $k^{\prime}>0$ and $t=0, W_{t}^{1}=\mathcal{L}_{z}(W)$ if $k^{\prime}=0$. If (ii) holds, set $t=t_{k^{\prime \prime}-1}, W_{t}^{1}=$ $\mathcal{L}_{f^{t}(z)}\left(W_{t_{k^{\prime \prime}}}\right)$ if $k^{\prime \prime}>0$ and $t=0, W_{t}^{1}=\mathcal{L}_{z}(W)$ if $k^{\prime \prime}=0$. If (iii) holds, set $t=t_{k^{*}}$ and $W_{t}^{1}=\mathcal{L}_{f^{t}(z)}(J)$. By the previous lemma, the required properties hold. 
In order to prove the claim we distinguish some cases.

Case I: $\phi: J \rightarrow W$ is monotone (so $k^{\prime}=k^{\prime \prime}$ ). For simplicity assume that $\phi$ is orientation preserving (otherwise consider the 2nd iterate of $\phi$ ) and $p$ a (the) fixed point of $\phi$. Let us introduce some notation: set $W_{-i}:=W_{t_{k-i}}, V_{-i}=V_{t_{k-i}}$. Let $W_{-i, \pm}$ be the components of $W_{-i} \backslash\{p\}$, labelled so that $W_{-i,+}$ are all on the same side of $p$ as $V$.

Case I.1: The union (of two adjacent fundamental neighbourhoods) $W_{0,+} \backslash$ $W_{-2,+}$ contains a $\sqrt{\xi^{\prime}}$-scaled neighbourhood of $V$. Then we are done (because their pullback has intersection multiplicity bounded by 3 ).

Case I.2: Case I.1 does not hold, but $\left|W_{-1,+}\right| W_{-2,+}\left|\geq \sqrt{\xi^{\prime}}\right| W_{-2,+} \mid$. Then $\left|W_{0,-}\right| \geq \frac{\xi^{\prime}-2 \sqrt{\xi^{\prime}}}{2 \sqrt{\xi^{\prime}}}\left|W_{-1,+}\right| \geq C_{1} \sqrt{\xi^{\prime}}\left|W_{-1,+}\right|$ (because $V$ is $\xi^{\prime}$-well-inside $W$ and Case I.1 does not hold). It follows that $V_{t_{i}}$ is $C \sqrt{\xi^{\prime}}$-well-inside $W$ for each $i=k^{\prime}, \ldots, k-1$. Using this statement for $i=k^{\prime}+1$, and pulling back once more, we get that $V_{t_{k^{\prime}}}$ is $\hat{\rho}\left(C_{1} \sqrt{\xi^{\prime}}\right)$-well-inside $J$.

Case I.3: $\left|W_{-1,+} \backslash W_{-2,+}\right| \leq \sqrt{\xi^{\prime}}\left|W_{-2,+}\right|$. Since $\phi: W_{-2,+} \rightarrow W_{-1,+}$ is quasipolynomial, this gives $\phi^{\prime} \leq C_{2} \sqrt{\xi^{\prime}}$ on $W_{-2,+}$. Hence, pulling back the external $\xi^{\prime}$-space of $V_{1}$ in $W_{-1}$, we get that $V_{-1}$ is $\rho_{1}\left(\xi^{\prime}\right)$-well inside $W_{-1,+} \backslash W_{-3,+}$. As in Case I.1 the claim follows.

Thus the first alternative in the claim holds in the monotone case.

Case II: $c$ is a turning point. If $c \notin W_{-2}$, the 3rd alternative holds, whereas if $c \in W_{-2}$ both components of $W_{-1} \backslash W_{-2}$ are comparable in length. So if $\mid W_{-1} \backslash$ $W_{-2}|\geq \sqrt{\xi}| W_{-2} \mid$, then $V_{t_{i}}$ is $\sqrt{\xi}$-well-inside $W_{-1}$ for $i=k^{\prime \prime}, \ldots, k^{\prime}$, and either the first or 3rd alternative of the claim holds. Otherwise $\left|W_{-1} \backslash W_{-2}\right| \leq \sqrt{\xi}\left|W_{-2}\right|$ and exactly as in Case I.3, $V_{-1}$ is very deep inside a component of $W_{-1} \backslash W_{-3}$. Since the intersection multiplicity of the pullback of this component along $f^{t_{k^{\prime \prime}}}(z), \ldots, f^{t_{k}}(z)$ is bounded by 3 (here we use that $W_{t_{k^{\prime \prime}}} \subset \cdots \subset W$ ), the 2 nd alternative of the claim holds.

Proposition 5. For each $\tilde{\xi}>0$ there exists $\xi>0$ such that if $I_{n+1}$ is $\xi$-well-inside $I_{n}$, then each return domain to $I_{n+1}$ is $\tilde{\xi}$-well-inside $I_{n+1}$.

Proof. Take $x \in I_{n+1}$, and let $t$ be its return time to $I_{n}$. Then choose the minimal $s>0$ such that $f^{s}(x)$ is in a return domain to $I_{n}$ which, for simplicity, we still denote by $I_{n+1}$, is $\xi$-well-inside $I_{n}$. Now let $W_{s}^{0}:=I_{n}$, and $V_{s}=I_{n+1}$, and let $W_{i}^{0}, V_{i}, i=0, \ldots, s$ be the pullbacks of $W_{s}^{0}$ and $V_{s}$ containing $f^{i}(x)$. Note that $V_{0}$ is the first return domain of $I_{n+1}$ containing $x$. Let $s_{1}^{\prime}$ be maximal with $0 \leq s_{1}^{\prime}<s$ and such that $W_{s_{1}^{\prime}}^{0}$ contains a critical point (if none of the intervals $W_{0}^{0}, \ldots, W_{s-1}^{0}$ contains a critical point, then set $s_{1}^{\prime}=0$ ). By part (2) of Theorem $\mathrm{C}$, there exists a function $\hat{\rho}$ with $\lim _{\xi \rightarrow \infty} \hat{\rho}(\xi)=\infty$ such that $W_{s_{1}^{\prime}}^{0}$ is a $\hat{\rho}(\xi)$-scaled neighbourhood of $V_{s_{1}^{\prime}}$. If $s_{1}^{\prime}=0$, then $x \in V_{0} \subset W_{0}^{0} \subset I_{n+1}$ and the proof is finished. If $s_{1}^{\prime}>0$, let $c_{1}^{\prime}$ be the critical point in $W_{s_{1}^{\prime}}^{0}$. Note that $W_{s_{1}^{\prime}}^{0} \supset V_{s_{1}^{\prime}}$ is also a nice pair. If the branch of the first return map to $W_{s_{1}^{\prime}}^{0}$ around $c_{1}$ is monotone, we set $c_{1}=c_{1}^{\prime}$ and $s_{1}:=s_{1}^{\prime}$. If it has a turning point, let $s_{1} \leq s_{1}^{\prime}$ be maximal for which $W_{s_{1}}^{0}$ contains a turning point $c_{1}$. Now let $t_{1}$ and $W_{t_{1}}^{1}$ be as in the previous lemma. So $V_{t_{1}}$ is $\rho_{1}(\hat{\rho}(\xi))$-well-inside $W_{t_{1}}^{1}$ and $W_{t_{1}}^{1} \supset V_{t_{1}}$ is a nice pair if $t_{1}>0$. If $t_{1}=0$, this completes the proof. If $t_{1}>0$, any pullback of $W_{t_{1}}^{1}$ that intersects $c_{1}$ is contained in $\mathcal{C}_{c_{1}}\left(W_{s_{1}}^{1}\right)$. Repeating this construction inductively, we get a sequence 
$t=t_{0} \geq s_{1}>t_{1}>\cdots>s_{\kappa-1}>t_{\kappa-1} \geq s_{\kappa} \geq t_{\kappa}=0$ and nice pairs $W_{t_{i}}^{i} \supset V_{t_{i}}$, $i=0,1, \ldots, \kappa-1$ (so that $W_{t_{i}}^{i}$ is a pullback of $I_{n}$ ), such that

(1) for $i=1, \ldots, \kappa, W_{s_{i}}^{i}$ is a $\hat{\rho}\left(\left(\rho_{1} \circ \hat{\rho}\right)^{i-1}(\xi)\right)$-scaled neighbourhood of $V_{s_{i}}$;

(2) for $i=0, \ldots, \kappa, W_{t_{i}}^{i}$ is a $\left(\rho_{1} \circ \hat{\rho}\right)^{i}(\xi)$-scaled neighbourhood of $V_{t_{i}}$;

(3) if $W_{t_{i}}^{i}, W_{t_{j}}^{j}(i<j)$ both contain the same critical point $c$, then $W_{t_{j}}^{j} \subset$ $\mathcal{C}_{c}\left(W_{t_{i}}^{i}\right)$.

Note that any pullback of $I_{n}$ intersecting $x$ is contained in $I_{n+1}$. Hence $I_{n+1}$ is a $(\rho \circ \hat{\rho})^{\kappa}(\xi)$-scaled neighbourhood of $V_{0}=\mathcal{L}_{x}\left(I_{n+1}\right)$. So if $\kappa$ is not large, the proposition immediately follows. If $\kappa$ is large, we will use a different argument: by the last property there is a critical point $c$, and a sequence $i(1)<i(2)<\cdots<i(r)$ (with $r \geq \kappa /(d-1)$ where $d$ is the number of critical points of $f$ ), such that

$$
W_{n_{i(1)}}^{i(1)} \supset \mathcal{C}_{c}\left(W_{n_{i(1)}}^{i(1)}\right) \supset W_{n_{i(2)}}^{i(2)} \supset \mathcal{C}_{c}\left(W_{n_{i(2)}}^{i(2)}\right) \supset W_{n_{i(3)}}^{i(3)} \supset \cdots \supset W_{n_{i(r)}}^{i(r)} \supset V_{n_{i(r)}} \cup\{c\} .
$$

From the first part of Theorem A, it follows that for $j=1, \ldots, r-1, \mathcal{C}_{c}\left(W_{n_{i(j+1)}}^{i(j+1)}\right)$ is $\delta$-well-inside $\mathcal{C}_{c}\left(W_{n_{i(j)}}^{i(j)}\right)$. By the first part of Theorem B, it follows that $\mathcal{L}_{x}\left(\mathcal{C}_{c}\left(W_{n_{i(j+1)}}^{i(j+1)}\right)\right)$ is $\delta^{\prime}$-well-inside $\mathcal{L}_{x}\left(\mathcal{C}_{c}\left(W_{n_{i(j)}}^{i(j)}\right)\right)$. Since this holds for $j=1, \ldots, r-$ $1, \mathcal{L}_{x}\left(W_{1}\right)$ contains a $\left(1+\delta^{\prime}\right)^{r-2}$-scaled neighbourhood of $\mathcal{L}_{x}\left(W_{n_{i_{r}}}\right)$. Note that $V_{0} \subset$ $\mathcal{L}_{x}\left(W_{n_{i_{r}}}\right)$ (it is the pullback of $V_{n_{i_{r}}} \subset W_{n_{i_{r}}}$ containing $x$ ) and that $\mathcal{L}_{x}\left(W_{n_{1}}\right) \subset I_{n+1}$ (because $W_{n_{1}}$ is a pullback of $\left.I_{n}\right)$. Hence $I_{n}$ is a $\left(1+\delta^{\prime}\right)^{r-2}$-scaled neighbourhood of $I_{n+1}=V_{0}$. This completes the argument if $\kappa$ (and therefore $r$ ) is large.

\section{Theorem D: Bounded Distortion}

Let us state here a more complete version of Theorem D.

Theorem $\mathbf{D}^{\prime}$ (Control of distortion). There is $\xi>0$ such that for any $\epsilon>0$ the following holds. Assume that $c_{1}$ is a recurrent critical point that is minimal w.r.t. the partial ordering $\prec$ on $\mathbf{C r}$, and let $c_{2}, \ldots, c_{k}$ be the collection of critical points with $\omega\left(c_{i}\right) \ni c_{1}, i=2, \ldots, k$. Then there exists a nice interval $W_{1} \ni c_{1}$ with $\left|W_{1}\right|<\epsilon$ such that $W_{i}:=\mathcal{L}_{c_{i}}\left(W_{1}\right)$ are pairwise disjoint nice intervals and $\mathrm{orb}^{+}(c) \cap W_{1}=\emptyset$ for any critical point $c \in \mathbf{C r} \backslash\left\{c_{1}, \ldots, c_{k}\right\}$. If $c_{i}$ is non-recurrent, then $W_{i} \cap \operatorname{orb}^{+}\left(c_{i}\right)=\emptyset$. If $f$ is infinitely renormalizable at $c_{1}$, then we can take for $W_{1}$ a periodic interval (with $f^{q}\left(\partial W_{1}\right) \subset \partial W_{1}$ where $q$ is the period of $W_{1}$ ). Denoting the first entry map to $W_{1}$ by $\psi$, the following properties hold.

(1) Let $V_{i} \ni c_{i}$ be the maximal interval such that $\psi\left(V_{i}\right) \subset W_{1}$ is contained in the return domain to $W_{1}$ containing $\psi\left(c_{i}\right)$, i.e., $V_{i}$ is maximal such that $\psi\left(V_{i}\right) \subset \mathcal{L}_{\psi\left(c_{i}\right)}\left(W_{1}\right)$. Then the first entry map $\Psi$ to $\bigcup V_{i}$ is a composition of at most $d$ maps of the type $L_{1} \circ f \circ L_{2}$, where $L_{1}, L_{2}$ are diffeomorphisms of bounded distortion. (Here $d$ is the number of critical points of $f$.)

(2) If $f$ is infinitely renormalizable of Feigenbaum type at $c_{1}$, then the period $q$ is of the form $q=p 2^{n}$. Let $V_{1} \subset W_{1}$ be the maximal interval containing $c_{1}$ with period $p 2^{n+1}$. For $i=2, \ldots, k$, let $V_{i} \ni c_{i}$ be the maximal interval such that $\psi\left(V_{i}\right) \subset \mathcal{L}_{\psi\left(c_{i}\right)}\left(V_{1}\right)$. Then again the first entry map to $\bigcup V_{i}$ is a composition of at most $d$ maps of the type $L_{1} \circ f \circ L_{2}$, where $L_{1}, L_{2}$ are diffeomorphisms of bounded distortion.

Proof. Let us first consider the case when $f$ is not Feigenbaum-like at $c_{1}$. Let $W_{1} \supset V_{1} \ni c_{1}$ be neighbourhoods of $c_{1}$ as in Theorem $\mathrm{A}^{\prime}$, case (2). Take $W_{1}$ so 
small that each critical point that does not accumulate on $c_{1}$ remains outside $W_{1}$. Then take $V_{i}, W_{i}$ as above, and let $s_{i} \geq 0$ be such that $\psi \mid W_{i}=f^{s_{i}}$. It follows that the intervals $W_{i}, \ldots, f^{s_{i}}\left(W_{i}\right)$ are pairwise disjoint and $V_{i}$ is well-inside $W_{i}$.

Now take $x \in M$ for which there exists the minimal $t \geq 0$ such that $f^{t}(x) \in \bigcup V_{j}$. So let us assume that $f^{t}(x) \in V_{j_{0}}$, and define the chains $\left\{\mathcal{V}_{i}\right\}_{i=0}^{t}$ and $\left\{\mathcal{W}_{i}\right\}_{i=0}^{t}$ such that $f^{i}(x) \in \mathcal{V}_{i} \subset \mathcal{W}_{i}, \mathcal{V}_{t}=V_{j_{0}}$ and $\mathcal{W}_{t}=W_{j_{0}}$. We claim that each critical point is contained in at most one of the intervals from $\left\{\mathcal{W}_{i}\right\}_{i=0}^{t}$. Indeed, if for $0 \leq i_{1}<i_{2} \leq t$ the intervals $\mathcal{W}_{i_{1}}$ and $\mathcal{W}_{i_{2}}$ contain the same critical point $c_{l}$, then $\mathcal{W}_{i_{1}} \subset \mathcal{W}_{i_{2}}$, and this would imply that $f^{i_{1}}(x) \in \mathcal{W}_{i_{1}} \subset V_{l}$, which contradicts the definition of $t$. Consider the intervals $\mathcal{W}_{n_{1}}, \ldots, \mathcal{W}_{n_{\nu}}$ from $\left\{\mathcal{W}_{i}\right\}_{i=0}^{t}$ that contain critical points. The previous theorem implies that (shrinking $W_{1}$ if necessary) the map $f_{\mid \mathcal{W}_{n_{j}+1}}^{n_{j+1}-n_{j}}$ (for $0 \leq j \leq \nu-1$, take $n_{0}=0$ ) has negative Schwarzian, and this implies that the diffeomorphism $f_{\mid \mathcal{V}_{n_{j}+1}}^{n_{j+1}-n_{j}-1}$ has bounded distortion. Hence, the first entry map to $\bigcup V_{i}$ is a composition of at most $d$ maps of the type $L_{1} \circ f \circ L_{2}$, where $L_{1}, L_{2}$ are diffeomorphisms of bounded distortion.

If $f$ is Feigenbaum-like at $c_{1}$, then let $W_{1} \ni c_{1}$ be a neighbourhood of $c_{1}$ as in Theorem $\mathrm{A}^{\prime}$, case 1, i.e., of period $p 2^{i}$. Because $W_{1}$ is periodic, $\mathcal{L}_{\psi\left(c_{i}\right)}\left(W_{1}\right)=W_{1}$ and so we cannot proceed precisely as above. But because of Corollary 1 (at the end of Section 21), for each $z \in W_{1}, \mathcal{L}_{z}\left(V_{1}\right)$ is well-inside $W_{1}$. With this modification, the proof goes as before.

Theorem D simply follows from Theorem $\mathrm{D}^{\prime}$.

\section{Theorem E: Measure of invariant Sets And ERGOdicity}

First let us prove the following result (which was proved previously in the negative Schwarzian unimodal case by Martens; see [14] and [17]).

Proposition 6. If $f(Y) \subset Y$ and $Y$ has positive Lebesgue measure, then either

- there exists an interval $H$ such that $|H|=|H \cap Y|$, or

- for almost every $y \in Y$ there exists a subset $C=C(y)$ of the critical set of $f$ such that $\Omega:=\omega(c)=\omega(\tilde{c})$ for each $c, \tilde{c} \in C$, each $c \in C$ is recurrent, $\Omega$ is a minimal set (every forward orbit is dense), $f^{n}(y) \rightarrow \Omega$ as $n \rightarrow \infty$, and $\lim _{n \rightarrow \infty} r_{n}(y) \rightarrow 0$.

Here $r_{n}(y)$ is defined as follows. Let $n$ be a positive integer, and let $T_{n}(y)$ be the maximal interval such that $f_{\mid T_{n}(y)}^{n}$ is a diffeomorphism. Then $r_{n}(y)$ is the length of the smallest component of $f^{n}\left(T_{n}(y) \backslash\{y\}\right)$.

Proof. Let us assume that the first alternative does not hold. Then $Y$ does not have full measure in any interval.

Claim 1: $y$ cannot accumulate onto parabolic or fixed points of $f$. To prove this we use an argument similar to the one given on pp. 482-483 of 9. More precisely, assume by contradiction that $y$ accumulates onto a parabolic or fixed point $a$. Let $a$ have period $s$, and for simplicity assume that $f^{s}$ is orientation preserving near $a$. Note that the period of parabolic points is bounded; see [17. Let $b \neq a$ be a periodic point for which there exists a sequence $b_{n}$ converging monotonically to $a$ (from the same side as $y, f(y), f^{2}(y), \ldots$ accumulates) so that $f^{s n}\left(b_{n}\right)=b$. Then $\left[b_{n}, b_{n+1}\right]$ are fundamental domains of the periodic point $a$. Fix $n_{0}$ large, let $f^{k}(y)$ be the first visit to $\left[b_{n_{0}}, a\right]$, and let $n$ be maximal such that 
$f^{k}(y) \in\left[b_{n}, b_{n+1}\right] \subset\left[b_{n}, a\right]$. If $n \geq n_{0}+1$, then the pullback of $\left[b_{n-1}, b_{n+2}\right] \supset$ $\left[b_{n}, b_{n+1}\right] \ni f^{k}(y)$ along $\left\{y, \ldots, f^{k}(y)\right\}$ is disjoint. So the pullback of $\left[b_{0}, b_{3}\right]$ along $\left\{y, \ldots, f^{k+s(n-1)}(y)\right\}$ has intersection multiplicity $\leq 3$. Since $\left[b_{1}, b_{2}\right] \ni f^{k+s(n-1)}(y)$ is well-inside $\left[b_{0}, b_{3}\right]$ (the sizes of adjacent intervals fundamental domains associated to $a$ are of the same order), by letting $n$ tend to infinity, and using that $y$ is a Lebesgue density point of $Y$, it follows that $Y$ contains either $\left[b_{0}, b_{1}\right]$ or $\left[b_{2}, b_{3}\right]$. (Note that the pullback of $\left[b_{0}, b_{3}\right]$ to $y$ might not be monotone, but that its image under $f^{k+s(n-1)}$ contains one of the components of $\left[b_{0}, b_{3}\right] \backslash f^{k+s(n-1)}(y)$.) So in this case, $Y$ contains an interval and the claim is proved by contradiction. If on the other hand, $n=n_{0}$, then $f^{k+s(n-1)}(y) \in\left[b, b_{1}\right]=\left[b_{0}, b_{1}\right]$, and then choose a sequence $U_{i}$ of adjacent fundamental domains associated to $b$ with $U_{i} \rightarrow b$ as $i \rightarrow \infty$ and with $b_{1} \in U_{0} \subset\left[b, b_{1}\right]$. If $f^{k+s(n-1)}(y) \in U_{0}$, then the previous argument can still be used to prove that $Y$ contains an interval (because $U_{0}$ is well-inside $\left[b_{0}, b_{3}\right]$ ). Otherwise let $m$ be maximal such that $y^{\prime}=f^{k+s(n-1)}(y) \in U_{m}$, and so by iterating $y^{\prime}$ to successive fundamental domains of $b$, there exists $k^{\prime}>k+s(n-1)$ with $f^{k^{\prime}}(y) \in U_{1}$. Now we can pull back $\left[U_{2}, U_{0}\right] \ni f^{k^{\prime}}(y)$ along $\left\{y, \ldots, f^{k^{\prime}}(y)\right\}$ with intersection multiplicity $\leq 3$ and as before obtain that $Y$ contains an interval, by letting $n$ tend to infinity. This contradiction concluded the proof of Claim 1 .

Claim 2: $r_{n}(y) \rightarrow 0$ for almost all $y \in Y$. (This fact is well known, but for completeness we prove it again.) Indeed, if this is not the case, then there are $\delta>0$, a subsequence $n_{i}, z \in Y$ and a Lebesgue density point $y$ of $Y$ with $r_{n_{i}}(y) \geq \delta$ and $f^{n_{i}}(y) \rightarrow z$. If the orbit of $y$ stays away from parabolic periodic points, then by part (2) of Theorem $\mathrm{C}$, and because $y$ is a Lebesgue density point and $Y$ is forward invariant, $Y$ contains (up to measure zero) a $\delta / 2$ neighbourhood of $z$, contradicting that $Y$ does not contain intervals.

Claim 3: Let $y$ be a point of Lebesgue density of $Y$. Then $\omega(y)=\bigcup_{c \in C} \omega(c)$ where $C=C(y)$ is the set of critical points such that $c \in \omega(y)$. By definition for each $n$ there is a critical point $c$ such that $f^{n}$ is a diffeomorphism on some interval $[a, y]$, such that $f^{k}(a)$ is a critical point $c$, and such that $f^{n-k}\left(c, f^{k}(y)\right)$ is a diffeomorphism. Since $r_{n}(y) \rightarrow 0$ and since $f$ has no wandering intervals, it follows that both $\left|c-f^{n}(y)\right|$ and $\left|f^{n-k}(c), f^{n}(y)\right|$ tend to zero when $n$ tends to infinity. Together this implies Claim 3.

Claim 4: If $y$ and $C=C(y)$ are as in Claim 3 and so that $y$ is not eventually mapped onto a critical point, then $c \in \omega\left(c^{\prime}\right)$ for any $c, c^{\prime} \in C$. This follows from the argument given in Proposition 3.3 of [9] (we use here that $Y$ does not have full measure in any interval). To prove this, let us first assume by contradiction that for some $c \in C$ there exists one or more critical points $c^{\prime} \in C$ such that $c \notin \omega\left(c^{\prime}\right)$. Let $C^{\prime}$ be the set of such critical points in $C$. Note that it is not impossible that $c \in C^{\prime}$. Define $\hat{C}=C \backslash C^{\prime}$. Let $U$ be a neighbourhood of $c$ such that each $c^{\prime} \in C^{\prime}$ avoids $U$. Let $I$ be a small nice interval, containing $c$ and well inside $U$, taken from the partition generated by the fixed points of $f$ and such that each first return domain to $I$ is well-inside $I$. This is possible by Theorem A. Now fix $I$ throughout the remainder of the proof of this claim and write $I(c)=I$. For each critical point $c^{*}$ such that $c \in \omega\left(c^{*}\right)$, let $k \geq 0$ be the smallest integer such that $f^{k}\left(c^{*}\right) \in I(c)$, and let $I\left(c^{*}\right)$ be the pullback of $I(c)$ along $\left\{c^{*}, \ldots, f^{k}\left(c^{*}\right)\right\}$. Then each return domain to $I\left(c^{*}\right)$ is also well-inside $I\left(c^{*}\right)$. Now fix some $c^{\prime} \in C^{\prime}$. Take arbitrarily small nice neighbourhoods $U_{j}$ of $c^{\prime}$ from the partition generated by the fixed points of $f$. We can take these neighbourhoods so that the forward orbit of their boundary points 
does not intersect $I(c)$ and if $k_{j} \geq 0$ is minimal with $f^{k_{j}}(y) \in U_{j}$, then $k_{j} \rightarrow \infty$. Let $n_{j}>k_{j}$ be minimal such that

$$
f^{n_{j}}(y) \text { is in a component } I(\tilde{c}) \text { of } \hat{I}=I(c) \cup \bigcup_{\hat{c} \in \hat{C}} I(\hat{c}) .
$$

Let $V_{j}^{0}, \ldots, V_{j}^{n_{j}}=I(\tilde{c})$ be the chain along $\left\{y, \ldots, f^{n_{j}}(y)\right\}$. Since $k_{j}$ is minimal and $I(\tilde{c})$ is nice, $V_{j}^{0}, \ldots, V_{j}^{k_{j}}$ are pairwise disjoint. Moreover, since the iterates of $c^{\prime} \in C^{\prime}$ avoid $U$, by the minimality of $n_{j}$, because $r_{n}(y) \rightarrow 0$ and the orbit of $y$ does not come close to critical points in the complement of $C=C(y), f^{n_{j}-k_{j}}: V_{j}^{k_{j}} \rightarrow \hat{I}$ is a diffeomorphism onto $I(\tilde{c})$. From Claim 1 we can assume that $\omega(y)$ does not contain fixed points of $f$ and so $f^{n_{j}}(y), j=1,2, \ldots$ are all contained in closed intervals $W^{\prime} \subset W \subset I(\tilde{c})$ which are all properly contained in each other. By assumption $Y$ does not have full Lebesgue measure in any subinterval, and therefore the density of $Y$ in $W$ is less than 1 . Since $f^{n_{j}}(y) \in W^{\prime}$, since $f^{n_{j}-k_{j}}: V_{j}^{k_{j}} \rightarrow I(\hat{c})$ is a diffeomorphism, and since $V_{j}, \ldots, V_{j}^{k_{j}}$ are disjoint, we get that the density of $Y$ in the pullback of $W$ to $y$ is also uniformly bounded away from 1 . This contradicts the assumption that $y$ is a Lebesgue density point of $Y$ and therefore proves the claim.

Claim 5: For $y$ as above, each $x \in \omega(y)$ accumulates on $C$. Indeed, assume not. Take an arbitrary $c \in C$ and take $I(c)=I_{n}$ as in the proof of Claim 4, so that the orbit of $x$ never enters the neighbourhood $I(c)$. Let $I\left(c^{*}\right), c^{*} \in C$, be the corresponding pullback of $I(c)$. Then take partition elements $U_{j}$ around $x$. Defining $k_{j}, n_{j}$ as in Claim 4 one gets again a contradiction.

Claim 6: Each point $x \in \omega(y)$ has an orbit that is dense in $\omega(y)$. Indeed, by the previous claim iterates of $x$ accumulate on some critical point $c \in C$. From Claim 4 , they then also accumulate on any other critical point, and so $\omega(x)=\bigcup_{c \in C} \omega(c)$. From Claim 2 the claim then follows.

From the previous claims the proposition follows.

Assuming that $X$ is a closed set that is forward invariant $(f(X) \subset X)$, define

$$
B(X)=\{y ; \omega(y) \subset X\} .
$$

Proposition 7. Let $X$ be a forward invariant set that is minimal. Then $X$ has zero Lebesgue measure and for each $x \in X$ there exists a sequence of intervals $\mathcal{N}_{n} \subset \mathcal{U}_{n}$ such that $\bigcap \mathcal{U}_{n}=\{x\},\left(\mathcal{U}_{n} \backslash \mathcal{N}_{n}\right) \cap X=\emptyset$ and $\mathcal{N}_{n}$ is well-inside $\mathcal{U}_{n}$. Moreover, if $Y \subset B(X)$ has positive Lebesgue measure, then $X$ is equal to $\omega(c)$ for at least one recurrent critical point $c$, and $\left|\mathcal{U}_{n} \cap Y\right| /\left|\mathcal{U}_{n}\right| \rightarrow 1$ as $n \rightarrow \infty$.

Proof. Take any point $x \in X$, and let $W_{1} \ni x$ be a nice interval such that all its return domains are well-inside. Let $V_{i} \subset W_{i}$ be the intervals given by Theorem $\mathrm{D}^{\prime}$, and let $\psi$ be the first entry map to $\bigcup V_{i}$. For a connected component $J$ of the domain of $\psi$, let $\psi(J) \subset V_{j_{0}}$ and $T(J) \supset J$ be the pullback of $W_{j_{0}}$. Because of the choice of intervals $V_{i} \subset W_{i}$, see Theorem $\mathrm{A}^{\prime}$, if $J_{1}, J_{2}$ are connected components of the domain of $\psi$ such that if $T\left(J_{1}\right) \cap T\left(J_{2}\right) \neq \emptyset$, then either $T\left(J_{1}\right) \subset T\left(J_{2}\right)$ or $T\left(J_{2}\right) \subset T\left(J_{1}\right)$. Moreover, if $J_{2} \cap T\left(J_{1}\right) \neq \emptyset$, then $T\left(J_{2}\right) \subset T\left(J_{1}\right)$. So let us take a minimal component $J$ in the sense that $X \cap J \neq \emptyset$ and such that $T(J) \subset T(\tilde{J})$ for any other component $\tilde{J}$ of $D(\psi)$ for which $X \cap \tilde{J} \neq \emptyset$. Since $X$ is compact this can be done. Because of this minimality, $T(J)$ contains at most $2^{b}$ components $\tilde{J}$ 
of $D(\psi)$ with $\tilde{J} \cap X \neq \emptyset$. Here $b$ denotes the number of turning points of $f$. Each one of these components $\tilde{J}$ is well-inside $T(J)$ and $T(\tilde{J})=T(J)$. It is not hard to see that this implies that there are intervals $N \subset U$ such that $U \subset T(J), N$ is well-inside $U$ and $X \cap(U \backslash N)=\emptyset$ and $N \cap X \neq \emptyset$. The choice for $N$ depends on the size and position of the components $\tilde{J}$ inside $T(J)$; it is not obvious that $N$ and $U$ are nice.

Since $X$ is minimal, for each $z \in X$ there exists the minimal $s \geq 0$ such that $f^{s}(z) \in U$. In fact, we have that $f^{s}(z) \in N$ because $X \cap(U \backslash N)=\emptyset$. Because $s$ is minimal, the pullback $z \in N_{s} \supset U_{s}$ of $N \supset U$ along $\left\{z, \ldots, f^{s}(z)\right\}$ is disjoint. So $N_{s}$ is well-inside $U_{s}$ and $X \cap\left(U_{s} \backslash N_{s}\right)=\emptyset$ (here we use Lemma 1). So, taking a sequence of $W_{1}$ shrinking to a point of $X$ we get a sequence $\mathcal{N}_{n} \subset \mathcal{U}_{n}$ as in the first statement.

From the previous proposition, it follows that if $Y \subset B(X)$ has positive Lebesgue measure and $X$ is minimal, then $X$ is equal to $\omega(\tilde{c})$ for at least one recurrent critical point $\tilde{c}$ (which is in $X$ ). Take $z=\tilde{c}$, let $\mathcal{U}_{n} \supset \mathcal{N}_{n} \ni \tilde{c}$ be as above, and let $C$ be the set of critical points $c$ with $\omega(c) \ni \tilde{c}$. Let $y$ be a Lebesgue density point of $Y$. From the previous proposition, there exists $t$ such that $f^{t}(y) \in \mathcal{N}_{n}$. Let $\mathcal{U}_{n}^{i} \supset \mathcal{N}_{n}^{i} \ni f^{i}(y), i=0, \ldots, t$ be the pullbacks of $\mathcal{U}_{n} \supset \mathcal{N}_{n}$ along $\left\{f^{i}(y), \ldots, f^{t}(y)\right\}$. From the properties of $\mathcal{U}_{n} \supset \mathcal{N}_{n}$ stated above, for $i<j \leq t$ with $\mathcal{U}_{n}^{i} \cap \mathcal{U}_{n}^{j} \neq \emptyset$ one either has $\mathcal{U}_{n}^{i} \subset \mathcal{U}_{n}^{j}$ or $\mathcal{U}_{n}^{i}$ is contained in a component of $\mathcal{U}_{n}^{j} \backslash \mathcal{N}_{n}^{j}$. Because of the minimality of $t$, the first case is impossible. Since $\mathcal{U}_{n} \cap X \subset \mathcal{N}_{n}$, for each critical point $c \in X$ there is at most one $i \leq t$ with $c \in \mathcal{N}_{n}^{i} \subset \mathcal{U}_{n}^{i}$. Moreover, $\mathcal{U}_{n}^{i} \cap X \subset \mathcal{N}_{n}^{i}$. Because of the contraction principle, provided $n$ is large, any critical point $c$ that is not in $X$ is not contained in any interval $\mathcal{U}_{n}^{i}$. From part (2) of Theorem $\mathrm{C}$ and since $y$ is a Lebesgue density point of $Y$, there exists $i(n) \leq t$ and a critical point $c \in X$ for which $c \in \mathcal{N}_{n}^{i(n)}$ such that $\left|Y \cap \mathcal{N}_{n}^{i(n)}\right| /\left|\mathcal{N}_{n}^{i(n)}\right|$ goes to one.

From the previous theorems and Propositions 6 and 7 Theorem E follows.

Remark 1. It is not true that any Cantor set $X$ with $f(X)=X$ has Lebesgue measure zero. Indeed, assume now that $\omega(c)$ is a Cantor set, and that its basin $B(\omega(c))$ has positive Lebesgue measure. We claim that in this situation, there is a Cantor set $X$ of positive Lebesgue measure with $X \subset B(\omega(c))$ and $f(X)=X$. (Examples of this situation are infinitely renormalizable maps, and non-renormalizable maps with an absorbing Cantor attractor; see [4].) To prove the claim, let $X_{0} \subset B(\omega(c))$ be a Cantor set of positive Lebesgue measure, and let $X_{1}$ be the closure of $\bigcup f^{i}\left(X_{0}\right)$. Then $f\left(X_{1}\right) \subset X_{1}$. Since $X_{0}$ is a Cantor set and $X_{0} \subset B(\omega(c))$, the set $X_{1}$ is closed, equal to $\bigcup f^{i}\left(X_{0}\right) \cup \omega(c)$, and $X_{1}$ has no isolated points. $X_{1}$ contains no intervals, because $B(\omega(c))$ contains no intervals. Hence $X_{1}$ is a Cantor set of positive Lebesgue measure with $f\left(X_{1}\right) \subset X_{1}$. In order to find a Cantor set with $f(X)=X$, take $X_{0}$ so that it lies in the range of $f$ and add appropriate preimages of $X_{1}$ to the set. For examples, if $f$ is unimodal, then we can define $X$ to be the union of $X_{1}$ and all $x$ so that for some integer $n \geq 1, x, \ldots, f^{n-1}(x)$ lies to the left of the critical point, while $f^{n}(x) \in X_{1}$. Again $X$ is a Cantor set of positive Lebesgue measure, but now $f(X)=X$.

\section{ACKNowledgement}

Most of this work was done when the second author was at the University of Warwick in 1999, and he is grateful for all the hospitality and support received 
there. Both authors were supported in part by EPSRC grant GR/R73171/01. The first version of this paper appeared in July 2000. The authors would like to thank Weixiao Shen for many useful discussions and for his remarks on the July 2001 version of this paper. On the request of the referee, we included Theorem B in July 2003. Theorem B was inspired by Weixiao Shen's big bounds (see [18. Theorem 1.4] and [20. Proposition 4.1]).

\section{REFERENCES}

[1] A. M. Blokh and M. Yu Lyubich, Measure and dimension of solenoidal attractors of onedimensional dynamical systems, Comm. Math. Phys. 127, (1990), 573-583. MR1040895 (91g:58164)

[2] A. M. Blokh and M.Yu. Lyubich, Measurable dynamics of S-unimodal maps of the interval, Ann. Sci. École Norm. Sup. 4e série, 24, (1991), 737-749. MR 1132757|(93f:58132)

[3] A.M. Blokh and M.Yu. Lyubich, Nonexistence of wandering intervals and structure of topological attractors of one-dimensional dynamical systems. II. The smooth case, Ergod. Th. Dyn. Sys., 9, (1989), 751-758. MR1036906 (91e:58101)

[4] H. Bruin, G. Keller, T. Nowicki and S. van Strien, Wild Cantor attractors exist, Annals of Math. 143, (1996), 97-130. MF1370759 (96m:58145)

[5] O. Kozlovskii, Axiom A maps are dense in the space of unimodal maps in the $C^{k}$ topology, Ann. of Math. 157 (2003), no. 1, 1-43. MR1954263 (2004b:37052)

[6] O. Kozlovski, Getting rid of the nagative Schwarzian derivative condition, Annals of Math. 152, (2000), 743-762. MF 1815700 (2002e:37050)

[7] O. Kozlovski, W. Shen and S. van Strien, Rigidity for real polynomials, Preprint 2003, available from http://maths.warwick.ac.uk/ strien/Publications

[8] G. Levin, Bounds for maps of an interval with one reflecting critical point. I, Fundamenta Math. 157, (1998), 287-298. MR1636895 (99g:58045)

[9] G. Levin and S. van Strien, Local connectivity of the Julia set of real polynomials, Annals of Math. 147, (1998), 471-541. MR 1637647 (99e:58143)

[10] G. Levin and S. van Strien, Bounds for maps of an interval with one reflecting critical point. II, Inventiones Math. 141, (2000), 399-465. MR 1775218|(2001i:37061)

[11] M.Yu. Lyubich, Non-existence of wandering intervals and structure of topological attractors of one dimensional dynamical systems: 1. The case of negative Schwarzian derivative, Ergod. Th. \& Dyn. Sys. 9, (1989), 737-749. MR1036905 (91e:58100)

[12] M.Yu. Lyubich, Ergodic theory for smooth one-dimensional dynamical systems, Stony Brook preprint 1991/11.

[13] R. Mañé, Hyperbolicity, Sinks and Measure in One Dimensional Dynamics, Commun. Math. Phys. 100, (1985), 495-524. MR0806250 (87f:58131)

[14] M. Martens, Interval dynamics, Thesis, Delft Technical University, (1990) and Distortion results and invariant Cantor sets of unimodal maps, Ergod. Th. Dynam. Sys., 14, 1994, 331-349. MR:1279474 (96c:58108)

[15] M. Martens, W. de Melo, and S. van Strien, Julia-Fatou-Sullivan theory for real onedimensional dynamics, Acta Math. 168, 1992, 273-318. MR.1161268|(93d:58137)

[16] W. de Melo and S. van Strien, A structure theorem in one-dimensional dynamics, Ann. of Math. (2), 129, 1989, 519-546. MR0997312 (90m:58106)

[17] W. de Melo and S. van Strien, One-Dimensional Dynamics, Ergebnisse der Mathematik und ihrer Grenzgebiete (3) 25, Springer Verlag, Berlin (1993). MR1239171 (95a:58035)

[18] W. Shen, Bounds for one-dimensional maps without inflection critical points, Preprint June 2000 and J. Math. Sci. Univ. Tokyo. 10, (2003), 41-88. MR.1963798

[19] W. Shen, On the measurable dynamics of real rational functions, Ergod. Th. Dyn. Sys. 23, 2003, 957-983. MR:1992673|(2004e:37069)

[20] W. Shen, On the metric properties of multimodal interval maps and $C^{2}$ density of Axiom A, Invent. Math. 156, 2004, 301-403. MR2052610

[21] G. Świątek and E. Vargas, Decay of geometry in the cubic family, Ergod. Th. \& Dyn. Sys. 18, (1998), 1311-1329. MR 1653256|(99h:58161) 
[22] S. van Strien, Hyperbolicity and invariant measures for general $C^{2}$ interval maps satisfying the Misiurewicz condition, Commun. Math. Phys. 128, (1990), 437-495. MR1045879 (91g:58161)

[23] E. Vargas, Measure of minimal sets of polymodal maps, Ergod. Th. and Dynam. Sys. 16, (1996), 159-178. MR1375131 (97a:58113)

Department of Mathematics, Warwick University, Coventry CV4 7AL, England

E-mail address: strien@maths.warwick.ac.uk

Department of Mathematics, University of São Paulo, São Paulo, Brazil

E-mail address: vargas@ime.usp.br 